\title{
12. MESSINIAN AND PRE-MESSINIAN SEDIMENTS FROM ODP LEG 107 SITES 652 AND 654 IN THE TYRRHENIAN SEA: SEDIMENTOLOGIC AND PETROGRAPHIC STUDY AND POSSIBLE COMPARISONS WITH ITALIAN SEQUENCES ${ }^{1}$
}

\author{
A. M. Borsetti, ${ }^{2}$ P. V. Curzi, ${ }^{3}$ V. Landuzzi, ${ }^{2}$ M. Mutti, ${ }^{4}$ F. Ricci Lucchi,${ }^{4}$ R. Sartori,${ }^{4}$ L. Tomadin, ${ }^{5}$ \\ and G. G. Zuffa ${ }^{4}$
}

\begin{abstract}
Sedimentology, mineralogy, and petrology of the pre-Pliocene sediments drilled at ODP Sites 652 and 654 in the Tyrrhenian Sea (Leg 107) have been studied with emphasis on the lower Messinian to pre-Messinian intervals.

Messinian at Site 652 is essentially turbiditic and basinal in character; it was deposited during the syn-rift phase in a strongly subsiding half-graben and is correlatable with emerged coeval sequences; in part with the Laga Formation of the foredeep of Apennines, and in part with the filling of grabens dissecting that chain in the Tyrrhenian portion of Tuscany.

The sequence found in Site 654 indicates an upper Tortonian to Messinian transgression accompanying crustal stretching in the western Tyrrhenian Sea and is perfectly correlatable with the so-called "Sahelian cycle" and with "postorogenic" cycles recognized in peninsular Italy and in Sicily.
\end{abstract}

\section{INTRODUCTION}

One of the main goals for ODP Leg 107 operations in the Tyrrhenian Sea was drilling through the Messinian evaporites and penetrating older sequences to reconstruct the history of subsidence and sedimentation in the basin. As is evident from the dense network of available seismic profiles (Moussat, 1983) and from the Site Survey performed in 1985 (Rehault et al., 1985), thick sequences of pre-evaporitic or non-evaporitic sediments occupy large domains throughout the east Sardinia passive type margin. The problem of drilling such sequences was addressed at ODP Sites 652 and 654 (Fig. 1).

This paper tries to characterize from a sedimentologic, petrographic, and stratigraphic point of view the recovered sequences, in order also to compare and possibly correlate them with coeval sequences cropping out in mainland Italy and in the major islands surrounding the Tyrrhenian Sea. The analytical studies have been mainly focused on the lowermost portions of the sequences, beneath the Messinian evaporites. However, the practical impossibility of placing stratigraphic boundaries at Site 652 and for the sake of completeness at Site 654 induced us to also take into account the bulk features of Messinian sedimentation.

\section{SITE 652}

\section{Location and Structural Setting}

The site is located on the lowermost Sardinia margin, at a water depth of some $3450 \mathrm{~m}$. It lies a few nautical miles east of an important structural feature, the R. Selli Line or Central Fault (Finetti and Del Ben, 1986; Sartori et al., 1987), across which a marked crustal thinning occurs, with shallowing of the MOHO to only $8-10 \mathrm{~km}$ in the vicinity of the site (Recq et al.,

\footnotetext{
${ }^{1}$ Kastens, K. A., Mascle, J., et al., 1990. Proc. ODP, Sci. Results, 107: College Station, TX (Ocean Drilling Program).

2 Istituto Geologia Marina CNR, Bologna, Italy.

${ }^{3}$ Dip. to Materiali Facolta Ingegneria, Universita, Ancona, Italy.

4 Dip. to Scienze Geologiche Universita, via Zamboni 67, Bologna, Italy.

5 Istituto Mineral. Petrogr., Universita Urbino, Italy.
}

1984). The boundary with oceanic crust occurs some nautical miles farther east. Site 652 was drilled on a tilted block a few kilometers wide, bounded by east-facing listric normal faults that strike almost north and accommodate basin stretching in the area. More information and details about the site are found in Kastens, Mascle, et al. (1987), from where most data presented below have been taken.

\section{Seismic stratigraphy}

Seismic profiles across the site show a geometry suggestive of pre-, syn-, and post-rift sequences. Five seismic units (S.U.) are recognized at the site location (Fig. 2).

S.U. I. $0-0.29$ s of penetration, interval velocity some 1.5 $\mathrm{km} / \mathrm{s}$. The unit is rather constantly transparent except for a few discontinuous high amplitude reflectors that show small unconformities and vertical displacements. A slight westward thickening is observed in the lower part. The bottom is represented by a series of high amplitude reflectors.

S.U. II. $0.29-0.42 \mathrm{~s}$ of penetration, interval velocity some 1.9 $\mathrm{km} / \mathrm{s}$. It is bounded by two sets of high amplitude reflectors, affected by small vertical displacements. Westward it thickens and changes acoustic characters.

S.U. III. $0.42-0.70 \mathrm{~s}$ of penetration, interval velocity some $2.6 \mathrm{~km} / \mathrm{s}$. It is bounded by high amplitude reflectors, with the top ones depicting irregular surfaces (erosional?). Westward, it thickens and changes in seismic character down to the depocenter of the half-garben, where possibly flowing evaporites (interval velocity some $3.4 \mathrm{~km} / \mathrm{s}$ ) are present.

S.U. IV. $0.70-0.90 \mathrm{~s}$ of penetration, interval velocity some $2.3 \mathrm{~km} / \mathrm{s}$. It contains discontinuous and high amplitude reflectors, suggesting either erosion or displaced sequences.

S.U. V. $0.90-1.14 \mathrm{~s}$ of penetration, interval velocity some 3.2 $\mathrm{km} / \mathrm{s}$. This unit is almost free of internal reflectors and is bounded at its base by an irregular surface, top of the true acoustic basement.

The general arrangement of these units suggests that most of S.U. I is post-rift, whereas its lower part, as well as S.U. II and III are syn-rift. S.U. IV and V are instead pre-rift in terms of development of the lower Sardinia margin. 


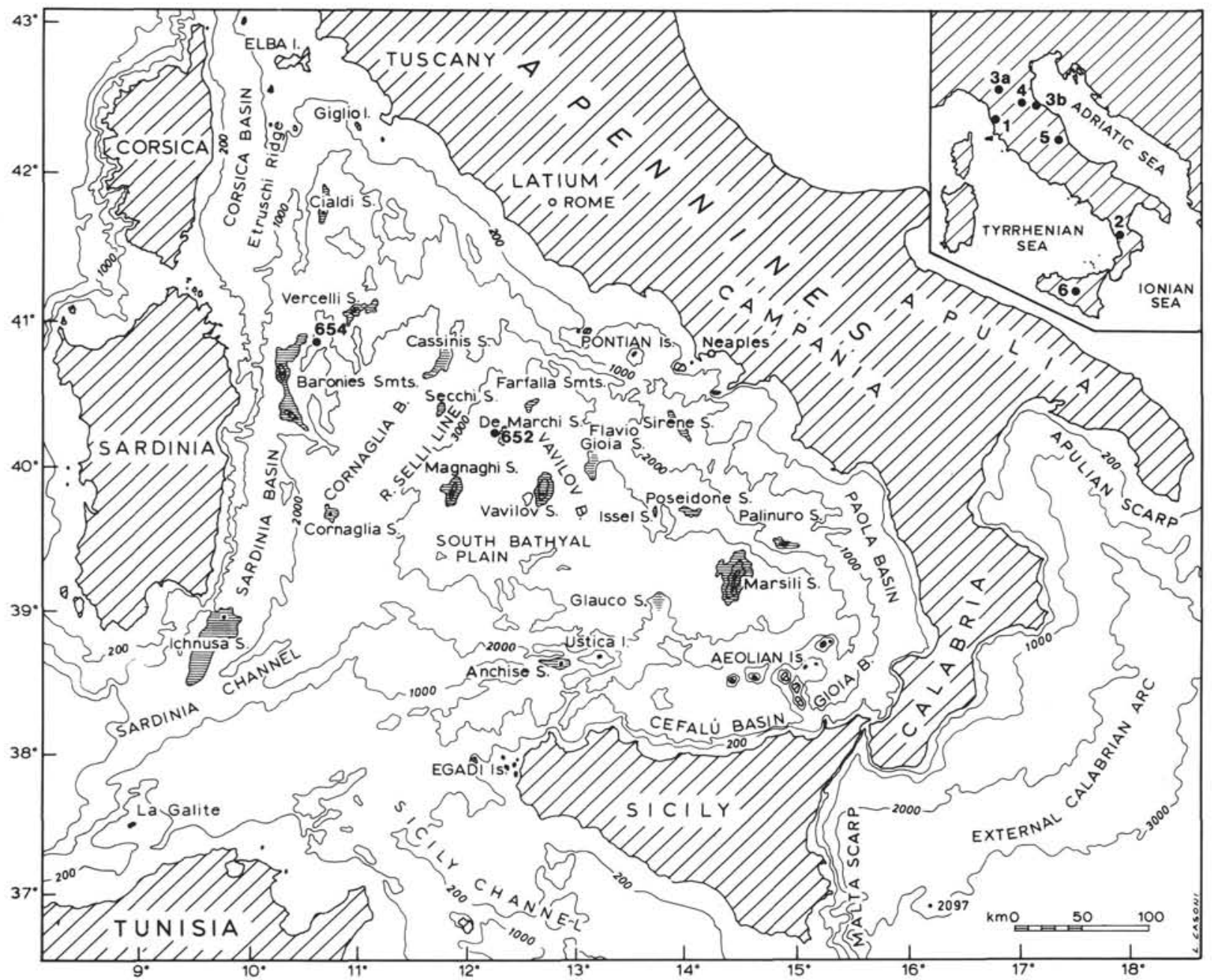

Figure 1. Schematic location of the studied sites in the Tyrrhenian Sea.

\section{Lithology and Stratigraphy}

At Site $652,721.1 \mathrm{~m}$ of sequence was drilled. Five major sedimentary units (subdivided into a few further subunits) were recognized (Fig. 2).

Unit 1. Cores 107-652-1R to 6R; 0-55.4 mbsf. Dominant muds and calcareous muds, with minor volcaniclastic component; age Pleistocene.

Unit 2. Cores 107-652A-7R to 20R-6, $50 \mathrm{~cm}$; 55.4-188.2 mbsf, thickness $132.8 \mathrm{~m}$. Dominant marly nannofossil oozes; age lower Pleistocene-Pliocene.

Unit 3. Cores 107-652A-20R-6, $50 \mathrm{~cm}$, to $20 \mathrm{R}-6,92 \mathrm{~cm}$; $188.2-$ $188.6 \mathrm{mbsf}$, thickness $0.42 \mathrm{~m}$. Strongly colored clays and muds; age top Messinian.

Unit 4. Cores 107-652A-20R-6, $92 \mathrm{~cm}$, to $36 \mathrm{R}$; 188.6-344.3 mbsf, thickness $155.7 \mathrm{~m}$. From 188.6 to 286 mbsf dominant turbidites made by sulfate- and carbonate-bearing sandy muds. From 286 to 335 mbsf same dominant lithology with abundant evidence of synsedimentary instability. From 335 to $344.3 \mathrm{mbsf}$ there is a polymict conglomerate with clasts typical of Mesozoic-Cenozoic suites of Southern Apennines or Sicily (Mascle, Sartori, et al., 1986). The age of the unit is probably Messinian, as also suggested by the presence of scarce Ammonia beccarii tepida and Cyprideis (both brackish water forms).

Unit 5. Cores 107-652A-37R to 75R; 344.3-721.1 mbsf, thickness (minimum) $376.8 \mathrm{~m}$. From 344.3 to 684 mbsf alternations of thin anhydrite- and carbonate-bearing sandy muds with cyclical interbedding of anhydrite intervals up to $5 \mathrm{~cm}$ thick. Minor lithologies include dark shales with plant debris, algae-rich intervals, carbon-rich layers, bright red and yellow millimeterthick horizons. From 684 to $721.1 \mathrm{mbsf}$, well indurated pelites and arenites, the latter becoming more coarse and common downward. The age, possibly Messinian, can not be determined due to the complete lack of in situ fossils. The only recognized forms are reworked Globigerinids (Tertiary), Morozovella (Paleocene), and Tintinnids (Jurassic-Early Cretaceous) inside rock fragments making up the arenites of the bottommost cores.

\section{Seismic and Lithologic Correlations (Fig. 2)}

S.U. I corresponds to Units 1, 2, 3, and to the upper part of Unit 4. Unit 1 (Pleistocene muds) is clearly post-rift; Unit 2 (lower Pleistocene to Pliocene nannofossil oozes) is mostly postrift and partly syn-rift. Unit 3 and part of Unit 4 (Messinian) are clearly and completely syn-rift. S.U. II, syn-rift, corresponds to most of Unit 4 (Messinian turbidites) and its bottom directly correlates with the conglomerate horizon at the base of Unit 4 .

S.U. III, syn-rift, and S.U. IV, pre-rift, correlate with Unit 5 (Messinian or pre-Messinian sandy muds and arenites) and are distinguishable only on the basis of a different degree of induration and of the frequency of coarse layers, that increases downward. The bottom hole correlates with the top of S.U. IV, that is, with the top of the pre-rift sequences. 


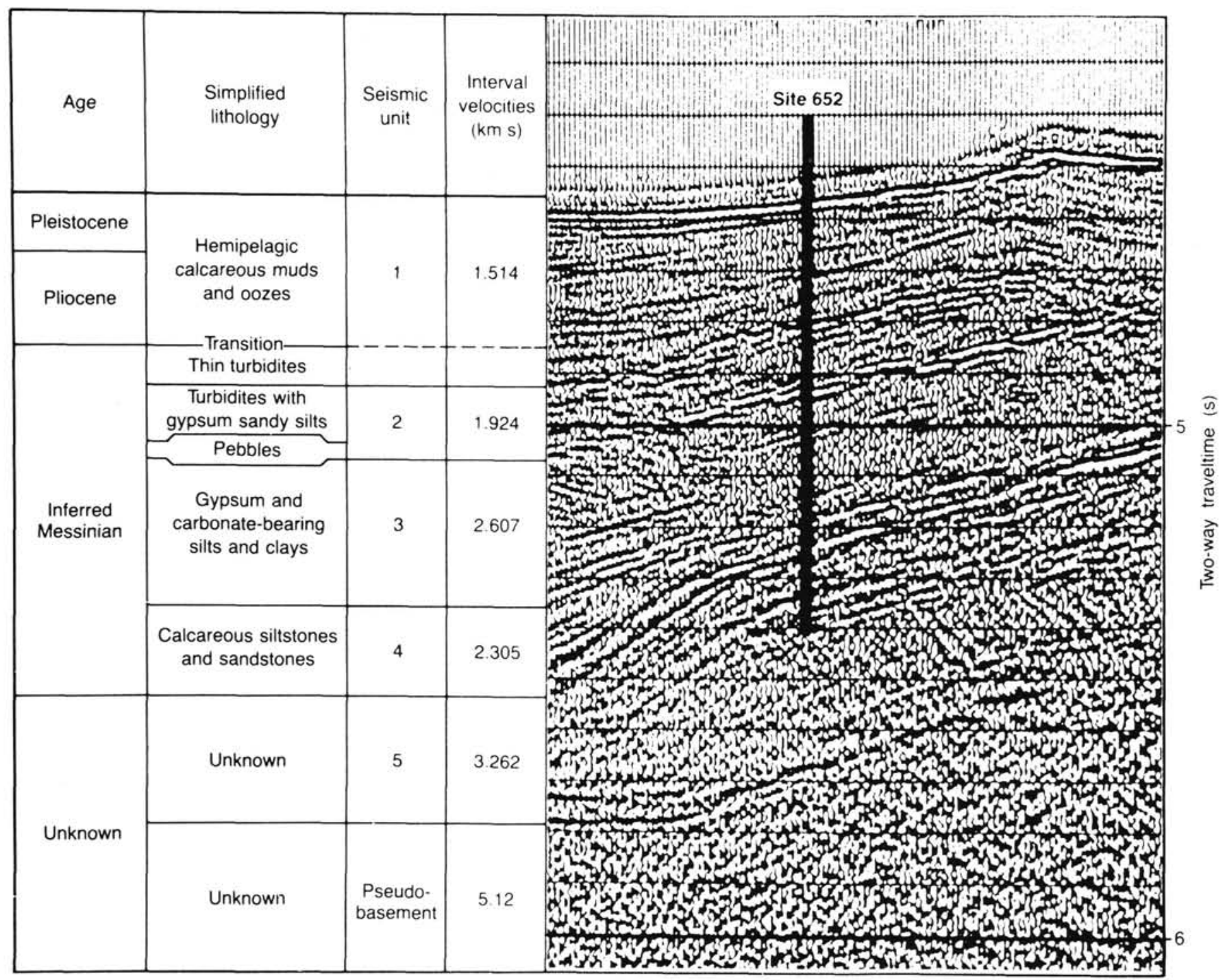

Figure 2. Seismic profile and schematic interpretation of the sequences drilled at Site 652 (from Kastens, Mascle, et al., 1987).

\section{Physical Sedimentology and Facies Analysis (Fig. 3)}

The about 540-m-thick pre-Pliocene succession is relatively uniform. The dominant facies is a closely spaced alternation of mud and silt. These couplets are the likely product of dilute, sluggish gravity flows (turbidity currents).

A vertical subdivision can be made on the basis of some marker horizons and lithofacies changes. Among the distinctive horizons:

1. The most prominent is a conglomerate body at some 334$345 \mathrm{mbsf}$;

2. The next marker downhole is an anhydrite-rich level in Core 44R (about $420 \mathrm{mbsf}$ ). Anhydrite is diffusely present in the interval 335-420 mbsf as white "laminae" or scattered nodules. The chosen marker represents the base of the interval and a maximum concentration of nodules;

3. Below $600 \mathrm{mbsf}$, some chaotic and contorted horizons suggest slumps; sandstone beds also occur in this section;

4 . Near the base of the section, a package of thicker calcareous sandstones displaying a coarsening-upward trend can be observed.
Among the facies changes we observe:

1. Certain characters change below and above the conglomerate. In the overlying part of the section, the color of the finer, muddy sediments is lighter, and the organic content seems correspondingly lower; the amount of recovery is less;

2. Anhydrite and dark shales are present essentially below the conglomerate; the sulfate is found, in discrete particles, over a thickness of about $80 \mathrm{~m}$; the shales, instead, become more frequent and dark downhole. Until Core 55R (525 mbsf), dark shales alternate with lighter muds, then become absolutely dominant. A transition between lighter and darker muds can be defined in the interval $500-530$ mbsf, where plastic deformation occurs (contorted levels $0.5-6 \mathrm{~m}$ thick);

3 . Regularly and thinly bedded dark shales with centimeterthick silt intercalations characterize the interval between 530 and $595 \mathrm{mbsf}$; no disturbed levels occur here;

4. Down to $690 \mathrm{mbsf}$, slumped and contorted beds are again frequent; they are sandwiched between the same shale-silt beds.

A basic rhythm of sedimentation can be observed both above and below the conglomerate body. The 1-10-cm-thick couplets 


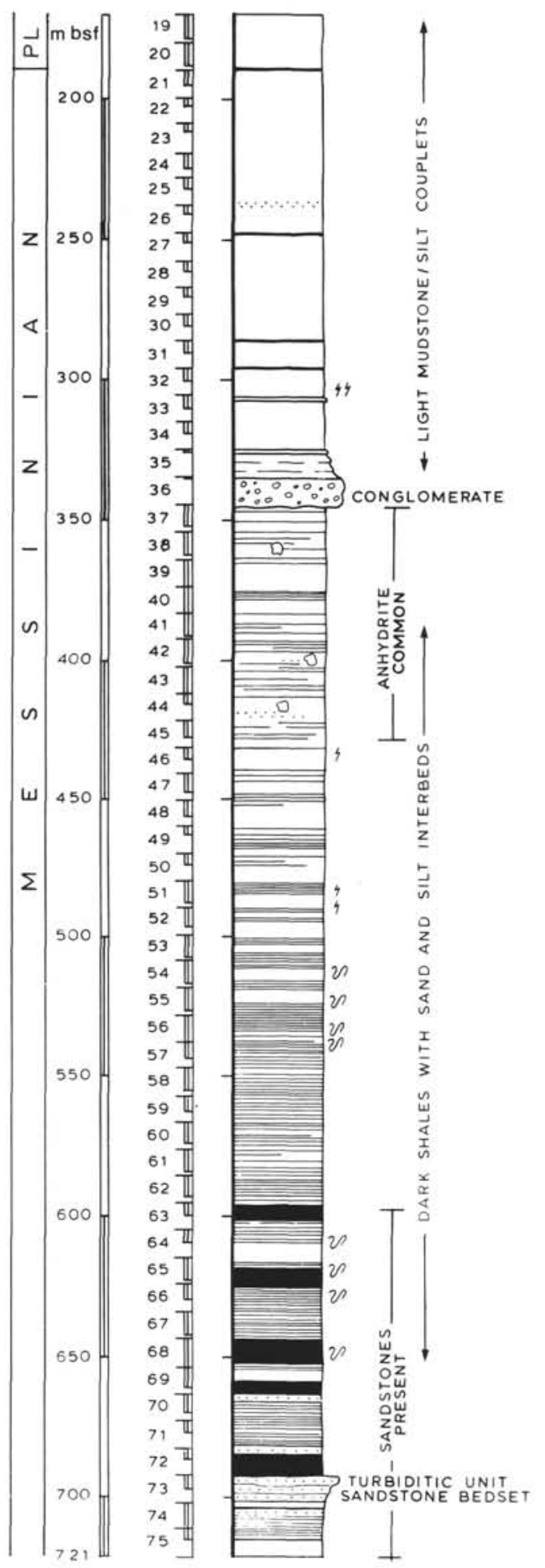

Figure 3. Recovery and schematic pre-Pliocene sequence in Site 652.

of silt and mud show the essential features of turbidites: sharp base, gradational top of silt, internal grading and/or lamination (plane-parallel to oblique and ripple-cross), and occasional scours at the base (e.g., Core 45R-1, 108-128 cm). Most couplets correspond to Bouma base-missing sequences.

Evidence of bioturbation is absent below the conglomerate, and rarely found above (vertical burrows). Evidence of mechan- ical reworking of beds by bottom currents (internal scours, winnowing of particles, etc.) is also lacking.

Summarizing, the section can be subdivided into the following parts or intervals, from bottom to top (Fig. 3):

1. Thin bedded turbidites and dark shales (including disseminated anhydrite) with a prominent sand body, $5 \mathrm{~m}$ thick, with base at some $700 \mathrm{mbsf}$, and several meter-thick contorted levels related probably to submarine slumps (from the bottom hole up to about $600 \mathrm{mbsf}$ );

2. Thin bedded silt-shale or sand-shale couplets with nodular/micronodular anhydrite scattered or aligned in millimeterthick "laminae"; some concentrations of nodules in massive to inversely graded units (from 422 to about $344 \mathrm{mbsf}$ );

3. Conglomerate;

4. Thin bedded silt-shale couplets $2-25 \mathrm{~cm}$ thick of relatively light color, with local bioturbation and sulfate clasts.

\section{Arenite Composition}

\section{Optical Analyses}

Quantitative optical analyses of arenites were performed on unstained thin sections: 400 points for each thin section were counted. Following the criteria proposed by Zuffa (1980, 1985, 1987), 55 compositional and textural classes were distinguished. These criteria are not revised in detail here but only a few specifications are reported.

When a coarse-grained rock fragment was encountered, both the type of crystal underneath the cross-hair and the type of rock fragment in which the crystal is contained were recorded (e.g., quartz in low-grade metamorphic fragment, Tables 1 and 2 ). This minimizes the dependence of arenite composition on grain size (Gazzi, 1966; Dickinson, 1970), while at the same time avoiding loss of information about the nature of coarse grained rock fragments. Two main groups of carbonate grains were distinguished: extrabasinal (terrigenous) grains and intrabasinal bioclastic grains. The latter are produced within the basin during the time interval of deposition of the studied sequence (CE and CI, respectively, in Tables 1 and 2). The criteria utilized in distinguishing the two groups are discussed in Zuffa (1985, 1987).

The framework of most of the examined samples has been extensively affected by carbonate replacements. In order to take into account these diagenetic modifications, suitable classes were introduced. The classes "carbonate replacement on quartz, Kfeldspar, plagioclase, and fine-grained lithic" were used when the replaced grain was still recognizable. When the original grain showed complete replacement by calcite, the class "carbonate replacement on undeterminable grain" was adopted. Finally the class "patchy carbonate" was introduced to indicate the fill of oversized pores formed by complete dissolution of framework grains.

\section{Results}

Nine samples were analyzed from the pre-Pliocene sequence. A first group of five are almost equally distributed within the bottommost cores of indurated sandstones (721.1-687.4 mbsf); the second group of four are from thin sandstone beds scattered within a pelitic sequence up to $607.4 \mathrm{mbsf}$. All these samples have a broadly similar composition, being composed of siliciclastic grains (NCE), most commonly fine-grained rock fragments of low-grade metamorphic rocks. Quartz is also common, whereas feldspars are rare. In addition to siliciclastic grains, the rock framework is made up of extrabasinal carbonate lithoclasts (CE), mostly micritic limestones which tend to disappear upsection. Some of them contain Tintinnid tests of Late Juras- 
Table 1. Modal point counts of Site 652 arenites.

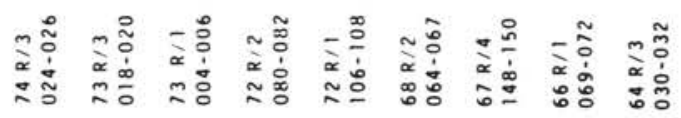

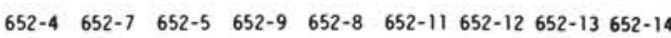

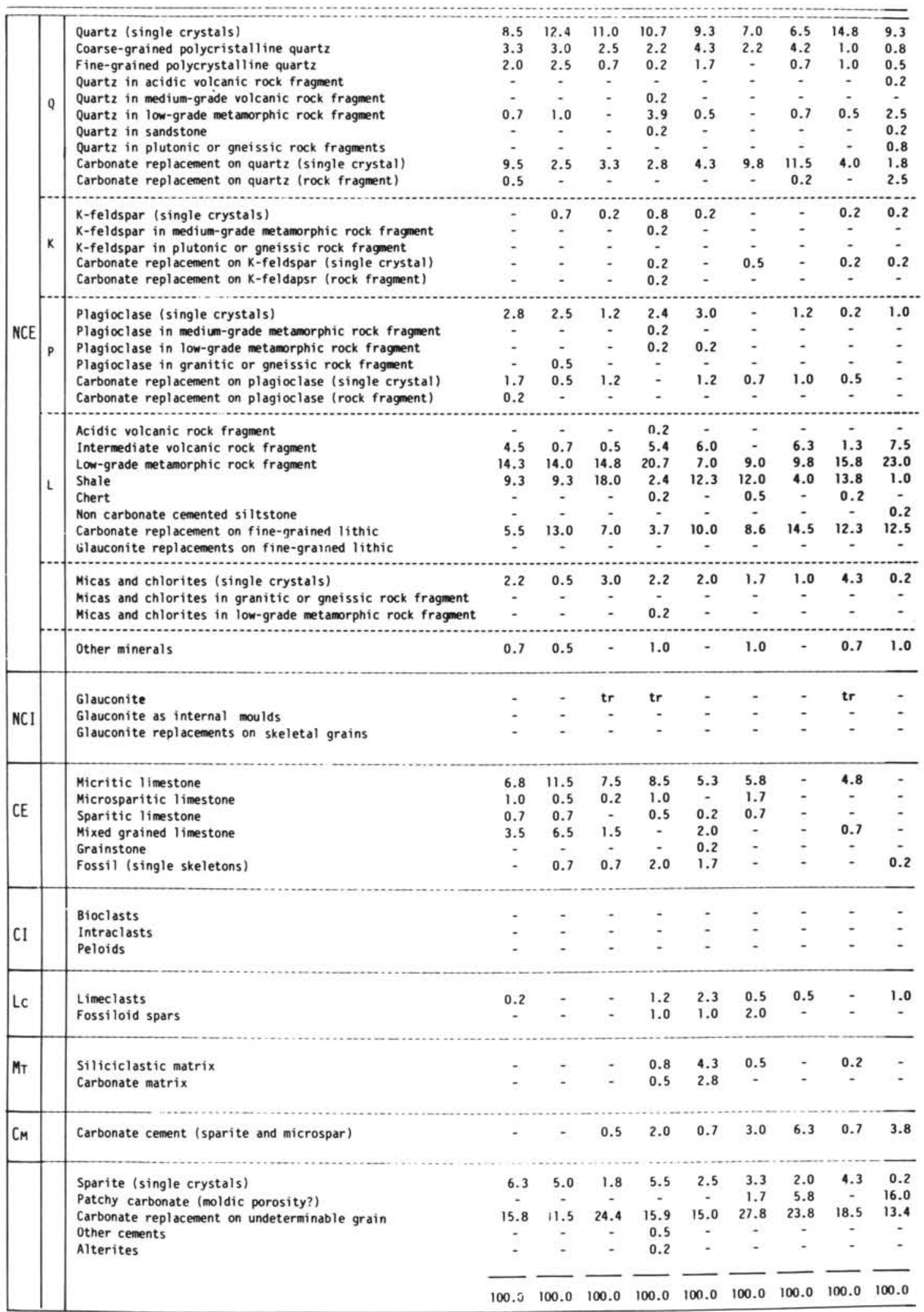


Table 2. Modal point counts of Site 654 arenites.

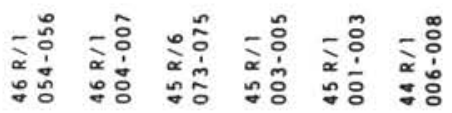

654-2 $\quad 654-1 \quad 654-5 \quad 654-4 \quad 654-3 \quad 654-6$

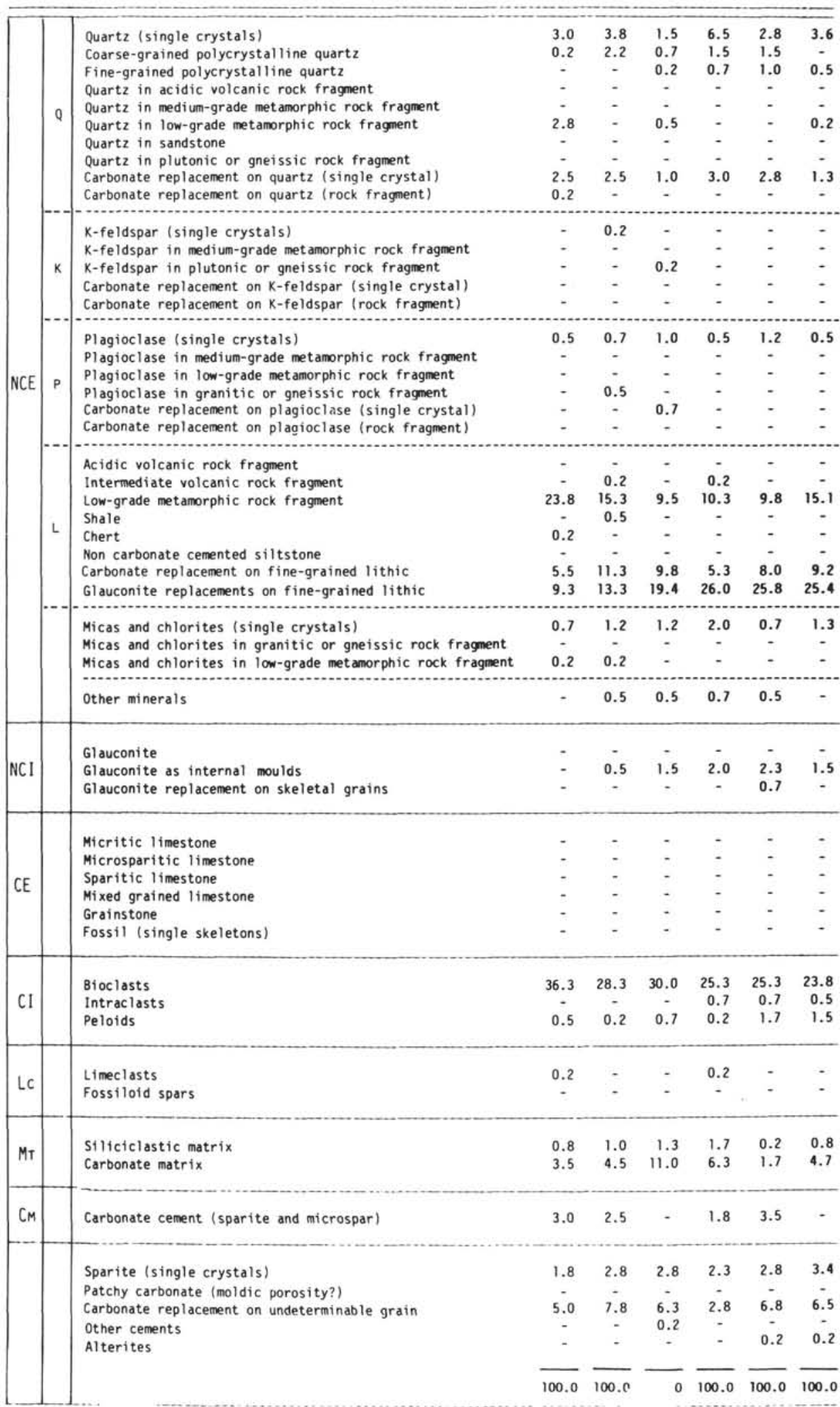


sic-Early Cretaceous age (Fig. 4B). Single bioclasts of planktonic foraminifers of Paleogene age are also present.

Interstitial calcite and dolomite are very common, but they seem more likely related to grain replacements rather than filling of pores.

Figure $5 \mathrm{~A}$ is a first-level classification and shows the main types of grains which make up the framework of these arenites. The QFL plot of Figure 5B shows that the NCE framework component can be classified in the field of litharenites. If carbonate lithoclasts were included in the "L group" this would shift the entire population of samples toward the $\mathrm{L}$ pole and their composition would become almost pure litharenite. The sand framework, although strongly modified by diagenetic processes (calcite and dolomite replacement of siliciclastic grains), indicates a hinterland source characterized by crystalline (mostly low-grade metamorphic rocks) and deep-water carbonate rocks

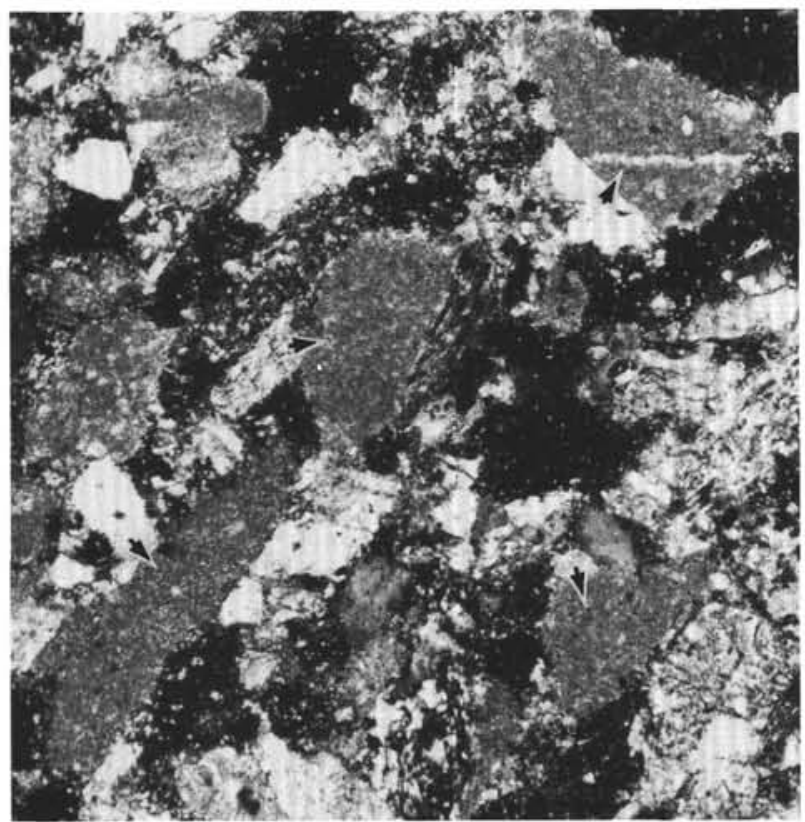

A

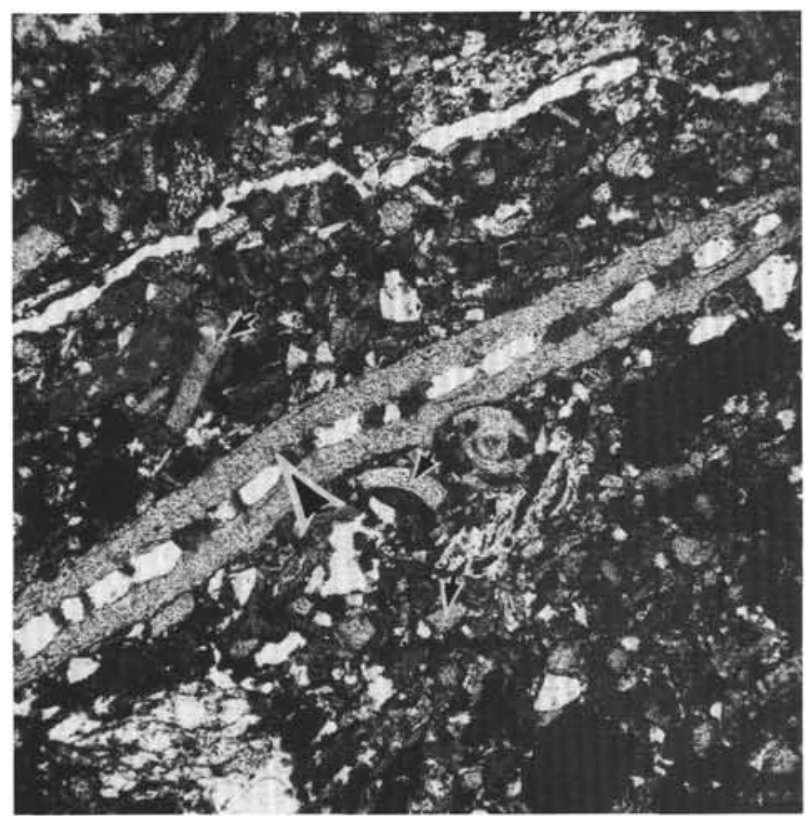

C
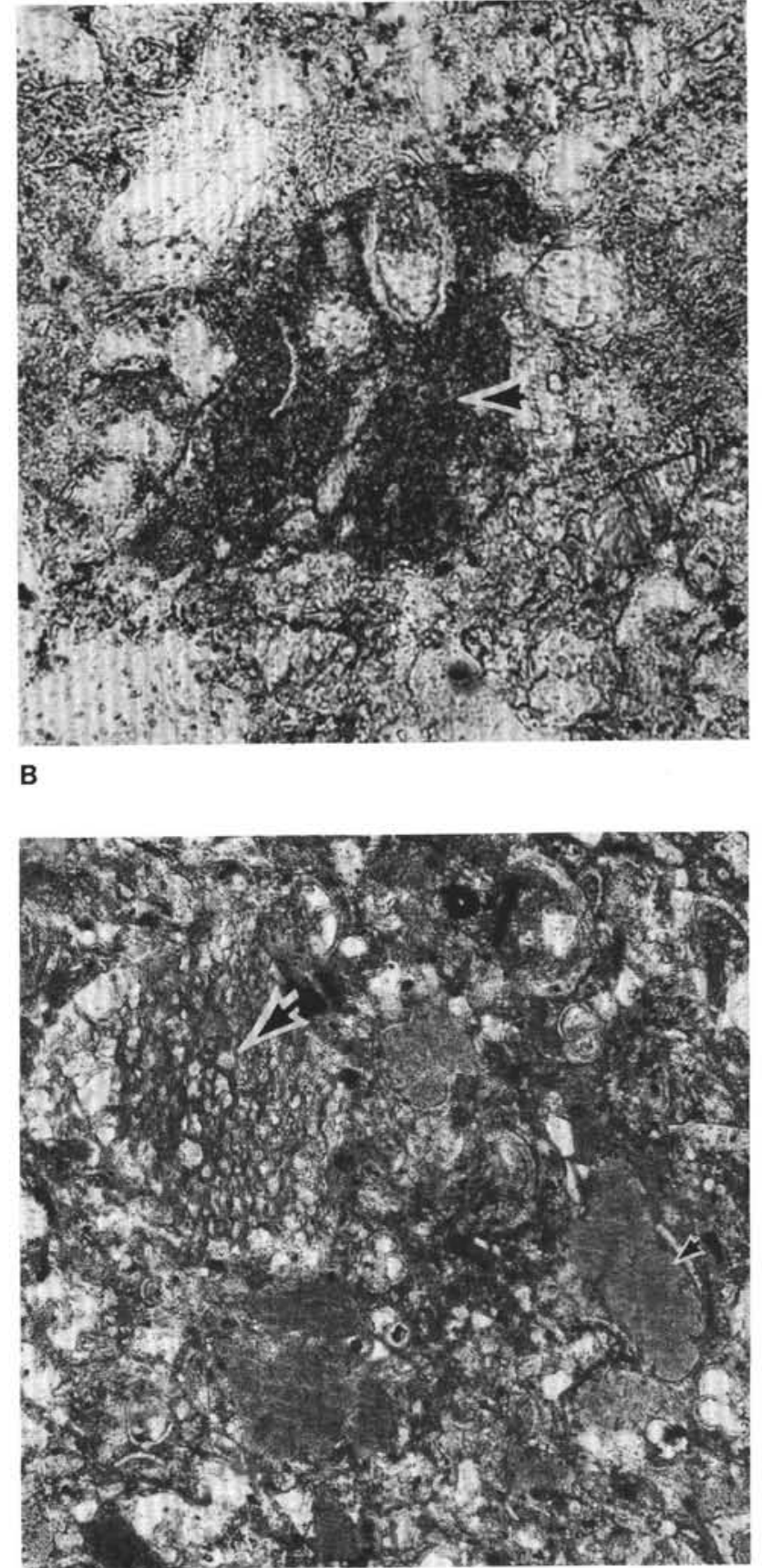

Figure 4. Examples of framework grains from arenites of Holes 652A and 654A. A. Terrigenous micritic lithoclasts (arrowed grains); the maximum diameter of the grain on the lower left is $0.4 \mathrm{~mm}$. Polarized light. Sample 652A-73R-3, 18-20 cm. B. Terrigenous micritic limestone with a Calpionellid test (Late Jurassic-Early Cretaceous): maximum diameter is $0.3 \mathrm{~mm}$. Polarized light, Site 652. C. Operculina (axial section): big arrow, maximum diameter is $4 \mathrm{~mm}$. Bioclasts of Operculina: small arrows. Polarized light. Sample 654A-46R-1, 54-56 cm. D. Glauconitized low-grade metamorphic rock fragment: big arrow, maximum diameter is $0.65 \mathrm{~mm}$. Bioclast with internal glauconitic mould: small arrow. Polarized light. Sample 654A-45R-6, $73-75 \mathrm{~cm}$. 


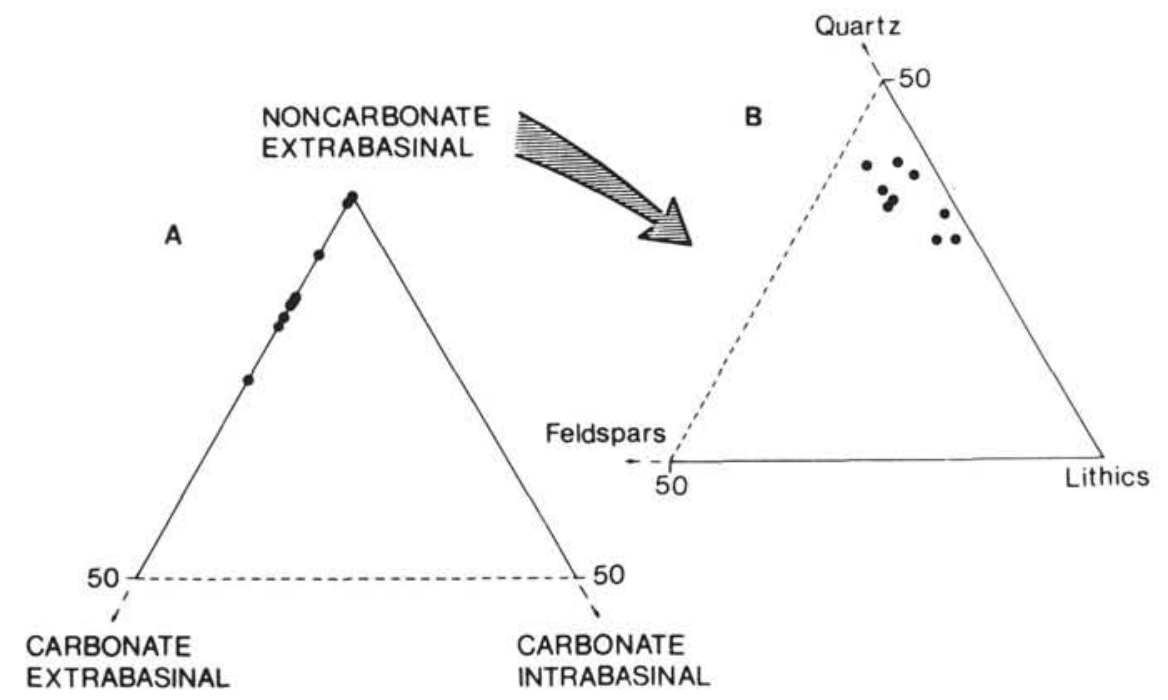

Figure 5. Framework composition of arenites from Site 652. A. Composition of main types of grains (first-level classification). B. Composition of the noncarbonate extrabasinal component (second-level classification).

of Cretaceous to Paleogene age. These carbonate grains are not derived from old platform-reef complexes. They are chiefly mudstone lithoclasts with sole pelagic fauna. Only very rare dolostone lithoclasts are present.

By taking into account regional geology and stratigraphy, a hinterland source area characterized by low-grade crystalline basement and by fine-grained deep-water carbonate turbidites (Sholle 1971a, b), for instance of the "Group" Helmintoides Flysch, can be envisaged. The small quantity of isolated fossils of Paleogene age can be possibly explained as reworking of foraminiferal tests contained in poorly lithified intervals of the carbonate turbidites.

\section{X-ray Diffraction Analyses}

\section{Methods}

Mineralogical analyses have been performed by x-ray diffraction both on bulk samples and on the clay fraction. The samples were dried and disaggregated with an agate mortar. The manual grinding produced a very fine powder suitable for analysis. Powder analyses were run with $\mathrm{Cu} \mathrm{K} \alpha$ (Ni filtered) radiation from $2^{\circ}$ to $70^{\circ}(2 \theta)$ at $1^{\circ} / \mathrm{min}(2 \theta)$ goniometer speed. After phase identification, mineral percentages were calculated following Cook et al. (1975).

The clay mineral composition in the $<2 \mu \mathrm{m}$ fraction was analyzed with the "smear on the glass" technique. Oriented samples, air dried at room temperature, glycolated, and heat-treated $\left(550^{\circ} \mathrm{C}\right)$, have been analyzed (Tomadin, 1974). The main claymineral groups recognized are illite, kaolinite, chlorite, smectite, and illite-smectite mixed-layers. A semiquantitative method (Biscaye, 1965) was used to evaluate the percentages of clay minerals, whose total amount is reported as recalculated to $100 \%$.

\section{Results}

We analyzed a large number of samples spanning from Core $37 \mathrm{R}$ to $75 \mathrm{R}$. The results, including the clay mineralogy, indicate a remarkable uniformity of sedimentation throughout the preconglomerate Messinian sequence (Table 3 ).

The clay mineral composition is rather constant and includes, in order of decreasing abundance: illite $(45 \%-60 \%)$, chlorite $(20 \%-30 \%)$, kaolinite $(10 \%-20 \%)$, smectite + illite-smectite $(8 \%-15 \%)$. Illite-smectite mixed-layers (with illite content predominant) are quite widespread throughout the $370 \mathrm{~m}$ of ex- amined sequence. A single deviation is found in the upper part of Core 51R, where chlorite suddenly increases to $50 \%-64 \%$, kaolinite disappears, and illite strongly decreases.

Powder analyses show that the anhydrite is present down to the bottom of the cored sequence. Background and normal amounts are in the order of $2 \%-10 \%$, with some laminae being constituted by pure anhydrite $(90 \%-100 \%)$. Apart from these latter cases, quartz content is always in the range $30 \%-40 \%$, while calcite content is also rather constant $(30 \%-40 \%)$ in Cores $37 \mathrm{R}-43 \mathrm{R}$. Below, calcite decreases to some $10 \%$, increasing again to about $30 \%$ from Core $62 \mathrm{R}$ downward. Dolomite is always present, with low amounts $(5 \%)$, in the upper part of the analyzed sequence. Downhole, it increases up to $30 \%-40 \%$ and up to $50 \%$ in levels alternating with others having the lower content seen in the overlying part. Below Core $71 \mathrm{R}$ the average content of dolomite is again low (some $5 \%$ ).

\section{Physical Properties of Sediments}

\section{Methods}

Samples of the pre-Pliocene sequences drilled were analyzed for evaluating their mechanical behavior on the basis of Atterberg indexes, following the method and classification reported by Chassefiere and Monaco (1983). Results of these authors apply to recent surficial sediments. We tried to observe whether the indexes could be valuable also in case of drilled sediments affected by strong burial and diagenesis. The indexes were measured by the British standard procedure using a cone test and distilled water. Grain size was determined with ASTM sieves and by photo-extinction sedimentometer. Bulk density, humidity, and undrained cohesion had already been measured onboard during drilling operations.

\section{Results}

Seven samples, encompassing more than $450 \mathrm{~m}$ of sequence in Units 4 and 5, were analyzed. Therefore, they only can give spot information about the alleged sedimentary environments of the units (Table 4).

The deepest studied sample (707.05 mbsf, base of Unit 5) is an inactive clayey silt (Skempton, 1953) that could be related to littoral or prodelta environments (Aloisi and Monaco, 1980). The overlying samples (Units 4 and 5) are normal active clayey silts (Skempton, 1953) typical of sediments accumulating, for 
Table 3. X-ray mineralogy of Site $\mathbf{6 5 2}$.

\begin{tabular}{|c|c|c|c|c|c|c|c|c|c|c|c|c|c|c|c|}
\hline \multicolumn{3}{|c|}{ Sample } & \multicolumn{9}{|c|}{ Mineral percentages (powders) } & \multicolumn{4}{|c|}{ Clay Minerals (100\%) } \\
\hline \&్મ & $\begin{array}{l}\text { ే } \\
\text { రુ } \\
\text { \& }\end{array}$ & 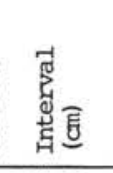 & $\begin{array}{l}\text { N̦ } \\
\text { 蛋 }\end{array}$ & 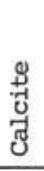 & $\begin{array}{l}9 \\
\stackrel{1}{E} \\
0 \\
8\end{array}$ & 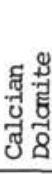 & 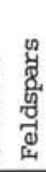 & $\begin{array}{l}\stackrel{8}{7} \\
\text { 苟 } \\
\text { 宾 }\end{array}$ & 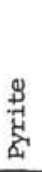 & $\begin{array}{l}9 \\
7 \\
8 \\
8\end{array}$ & 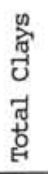 & 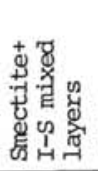 & $\begin{array}{l}\underset{8}{8} \\
\underset{H}{7}\end{array}$ & 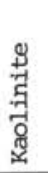 & 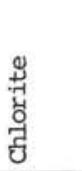 \\
\hline 37 & 2 & $94-96$ & 22 & 24 & 10 & 12 & 7 & 15 & - & - & 9 & & & & \\
\hline 37 & 3 & $80-82$ & 44 & 22 & 5 & - & 6 & 3 & - & - & 17 & 10 & 48 & 20 & 22 \\
\hline 37 & 51 & $133-135$ & 31 & 32 & 3 & 3 & 8 & 7 & - & - & 14 & 9 & 39 & 24 & 28 \\
\hline 37 & 6 & $6-8$ & 33 & 32 & - & 5 & 10 & 3 & - & - & 15 & 14 & 44 & 20 & 22 \\
\hline 38 & 1 & $21-23$ & 35 & 30 & 4 & 4 & 7 & - & - & - & 18 & 9 & 47 & 20 & 24 \\
\hline 38 & 3 & $87-89$ & 10 & 11 & - & - & 3 & 64 & - & - & 8 & & & & \\
\hline 38 & 3 & $111-115$ & - & - & - & - & - & 100 & - & - & - & & & & \\
\hline 38 & 5 & 9-12 & 28 & 28 & 4 & 4 & 6 & 13 & - & - & 15 & 10 & 47 & 20 & 23 \\
\hline 38 & 5 & $79-81$ & 35 & 35 & 2 & 4 & 6 & - & - & - & 16 & 10 & 47 & 21 & 22 \\
\hline 39 & 1 & $26-29$ & 35 & 36 & 3 & 4 & 5 & - & - & - & 15 & 9 & 46 & 22 & 23 \\
\hline 39 & 11 & $129-131$ & 34 & 31 & 4 & 4 & 9 & - & - & - & 16 & 10 & 46 & 22 & 22 \\
\hline 39 & 4 & $66-68$ & 35 & 33 & 3 & 4 & 8 & - & - & - & 16 & 10 & 46 & 20 & 24 \\
\hline 39 & 7 & $6-8$ & 31 & 34 & 3 & 4 & 10 & - & - & - & 16 & 10 & 42 & 23 & 25 \\
\hline 40 & 1 & $72-74$ & 34 & 34 & 3 & 3 & 5 & - & - & - & 19 & 9 & 50 & 18 & 23 \\
\hline 40 & 31 & $117-119$ & 33 & 34 & 2 & 3 & 9 & 2 & - & - & 14 & 9 & 46 & 21 & 24 \\
\hline 40 & 6 & $60-62$ & 35 & 36 & 3 & 4 & 5 & - & - & - & 15 & 11 & 43 & 21 & 25 \\
\hline 40 & 7 & $33-35$ & 34 & 31 & 3 & 3 & 10 & - & - & - & 16 & 9 & 47 & 19 & 25 \\
\hline 41 & 1 & $72-74$ & 38 & 32 & 3 & 3 & 7 & - & - & - & 17 & 10 & 48 & 20 & 22 \\
\hline 41 & 2 & $50-52$ & 36 & 35 & - & 4 & 5 & - & - & - & 18 & 8 & 47 & 21 & 24 \\
\hline 41 & 3 & $136-138$ & 23 & 19 & - & 2 & 4 & 36 & - & - & 14 & 8 & 49 & 20 & 23 \\
\hline 41 & 6 & $30-33$ & 32 & 34 & 3 & 4 & 7 & - & - & - & 17 & 10 & 46 & 20 & 24 \\
\hline 42 & 2 & $24-27$ & 37 & 32 & 4 & 3 & 6 & - & - & - & 16 & 12 & 47 & 19 & 22 \\
\hline 42 & 5 & $89-91$ & 33 & 34 & 3 & 4 & 5 & - & - & - & 19 & 10 & 44 & 22 & 24 \\
\hline 42 & 5 & $144-146$ & 36 & 32 & 3 & 4 & 6 & - & - & - & 17 & 9 & 48 & 20 & 23 \\
\hline 42 & 6 & 53-55 & 7 & 10 & 2 & 3 & 2 & 70 & - & - & 6 & & & & \\
\hline 43 & 4 & $38-40$ & 23 & 30 & 5 & 9 & 10 & 16 & - & - & 7 & & & & \\
\hline 43 & 4 & $41-43$ & 25 & 28 & 5 & 13 & 6 & 13 & - & - & 10 & & & & \\
\hline 43 & 4 & $48-50$ & 36 & 33 & 4 & 4 & 5 & - & - & - & 16 & 9 & 48 & 22 & 21 \\
\hline 43 & 5 & $93-96$ & 30 & 35 & 4 & 6 & 5 & - & - & - & 18 & 9 & 48 & 20 & 23 \\
\hline 44 & 1 & $96-99$ & 33 & 33 & 3 & 2 & 7 & - & - & - & 19 & 10 & 46 & 22 & 22 \\
\hline 44 & 2 & $66-68$ & 37 & 27 & 4 & 4 & 5 & - & - & - & 21 & 9 & 51 & 17 & 23 \\
\hline 44 & 3 & $75-77$ & 8 & 11 & 4 & 3 & 2 & 67 & - & - & 5 & & & & \\
\hline 44 & $\mathrm{CC}$ & $4-6$ & 1 & - & - & - & - & 99 & - & - & - & & & & \\
\hline 45 & 1 & $137-139$ & 33 & 38 & 3 & 8 & 8 & - & - & - & 9 & & & & \\
\hline 45 & 2 & $62-64$ & 40 & 25 & 3 & 5 & 3 & - & - & - & 21 & 11 & 49 & 19 & 21 \\
\hline 45 & 3 & $78-82$ & 36 & 31 & 5 & 11 & 5 & - & - & - & 11 & & & & \\
\hline 45 & 3 & $96-103$ & 40 & 24 & - & 5 & 4 & - & - & - & 23 & 10 & 46 & 22 & 22 \\
\hline 46 & 1 & $60-62$ & 34 & 33 & 5 & - & 7 & 1 & - & - & 18 & 14 & 52 & 18 & 16 \\
\hline 46 & 2 & $71-73$ & 36 & 33 & 2 & - & 6 & 2 & - & - & 19 & 15 & 55 & 15 & 17 \\
\hline 46 & 2 & $115-117$ & 32 & 33 & 4 & 8 & 9 & - & - & - & 13 & 12 & 47 & 23 & 18 \\
\hline 46 & 3 & $100-102$ & 38 & 31 & 3 & - & 6 & - & - & - & 18 & 13 & 50 & 21 & 16 \\
\hline 47 & 1 & $64-66$ & 29 & 29 & 5 & 3 & 16 & - & - & - & 16 & 11 & 50 & 20 & 19 \\
\hline 47 & 1 & $126-128$ & 36 & 26 & 4 & - & 9 & 1 & - & - & 22 & 12 & 56 & 14 & 18 \\
\hline 47 & 4 & $54-56$ & 30 & - & 48 & - & 14 & 1 & - & - & 6 & & & & \\
\hline 47 & 5 & $32-34$ & 36 & 30 & 5 & 8 & 8 & 1 & - & - & 12 & 11 & 51 & 18 & 20 \\
\hline 48 & 1 & $8-10$ & 37 & 36 & 3 & - & 9 & 2 & - & - & 12 & 11 & 56 & 15 & 18 \\
\hline 48 & 1 & $85-88$ & 34 & 34 & 2 & - & 10 & 1 & - & - & 17 & 12 & 56 & 17 & 15 \\
\hline 48 & 3 & $51-54$ & 37 & 32 & 2 & - & 4 & 3 & - & - & 20 & 10 & 60 & 13 & 17 \\
\hline 48 & 3 & $130-133$ & 33 & 34 & 2 & - & 3 & 3 & - & - & 22 & 12 & 55 & 15 & 18 \\
\hline 49 & 1 & $23-25$ & 41 & - & 23 & - & 12 & 8 & - & - & 13 & 14 & 53 & 16 & 17 \\
\hline 49 & 1 & $65-67$ & 30 & 33 & 13 & - & 17 & tr & - & - & 6 & & & & \\
\hline 49 & 1 & $140-142$ & 34 & 27 & 6 & - & 8 & 2 & - & - & 21 & 11 & 54 & 17 & 18 \\
\hline 49 & 2 & $95-97$ & 26 & - & 48 & - & 16 & 1 & - & - & 8 & & & & \\
\hline 50 & 1 & $59-62$ & 50 & - & 11 & - & 9 & 2 & - & - & 25 & 13 & 54 & 16 & 17 \\
\hline 50 & 2 & $61-63$ & 36 & - & 39 & - & 8 & 1 & - & - & 14 & & & & \\
\hline 50 & 2 & $90-103$ & 40 & 19 & 4 & - & 10 & - & - & - & 24 & 14 & 44 & 17 & 25 \\
\hline 50 & 3 & $9-11$ & 39 & - & 35 & - & 11 & 1 & - & - & 12 & & & & \\
\hline 50 & $\propto$ & $13-15$ & 38 & 22 & 3 & - & 11 & 4 & - & - & 20 & 10 & 55 & 15 & 20 \\
\hline 51 & 1 & $13-15$ & 34 & 43 & 3 & - & 8 & - & - & - & 11 & 8 & 28 & - & 64 \\
\hline 51 & 1 & $106-108$ & 38 & 27 & 4 & - & 6 & 2 & - & - & 21 & 14 & 35 & - & 51 \\
\hline 51 & 3 & $128-130$ & 47 & 25 & 4 & - & 4 & 3 & - & - & 17 & 11 & 46 & 16 & 27 \\
\hline 51 & 5 & $126-128$ & 38 & 40 & 3 & - & 8 & 1 & - & - & 8 & & & & \\
\hline 51 & 6 & $44-46$ & 43 & - & 31 & - & 3 & 1 & - & - & 19 & 13 & 53 & 15 & 19 \\
\hline 52 & 1 & $53-54$ & 36 & - & 32 & - & 9 & 1 & - & - & 19 & & & & \\
\hline 52 & 2 & $50-52$ & 45 & - & 21 & - & 5 & 2 & - & - & 23 & 11 & 59 & 13 & 17 \\
\hline 52 & 2 & $78-80$ & 38 & 33 & 3 & - & 5 & - & - & - & 19 & 11 & 50 & 19 & 20 \\
\hline 52 & 3 & $138-140$ & 40 & - & 31 & - & 4 & 1 & - & - & 21 & 10 & 53 & 17 & 20 \\
\hline 52 & 4 & $67-69$ & 35 & 34 & 7 & - & 2 & 4 & - & - & 16 & 11 & 57 & 13 & 19 \\
\hline 53 & 1 & $72-74$ & 37 & - & 30 & - & 3 & 2 & - & - & 25 & 12 & 53 & 16 & 19 \\
\hline 53 & 3 & $48-50$ & 38 & 40 & 4 & - & 4 & 1 & - & - & 12 & 11 & 54 & 16 & 19 \\
\hline 53 & 3 & $146-148$ & 39 & - & 29 & - & 4 & 1 & - & - & 24 & 12 & 55 & 15 & 18 \\
\hline 53 & 4 & $14-16$ & 35 & - & 38 & - & 3 & 1 & - & - & 21 & 12 & 53 & 18 & 17 \\
\hline 54 & 1 & $96-98$ & 33 & - & - & 31 & 3 & 3 & - & - & 27 & 13 & 54 & 15 & 18 \\
\hline
\end{tabular}


Table 3 (continued).

Sample Mineral percentages (powders) Clay Minerals (100\%)

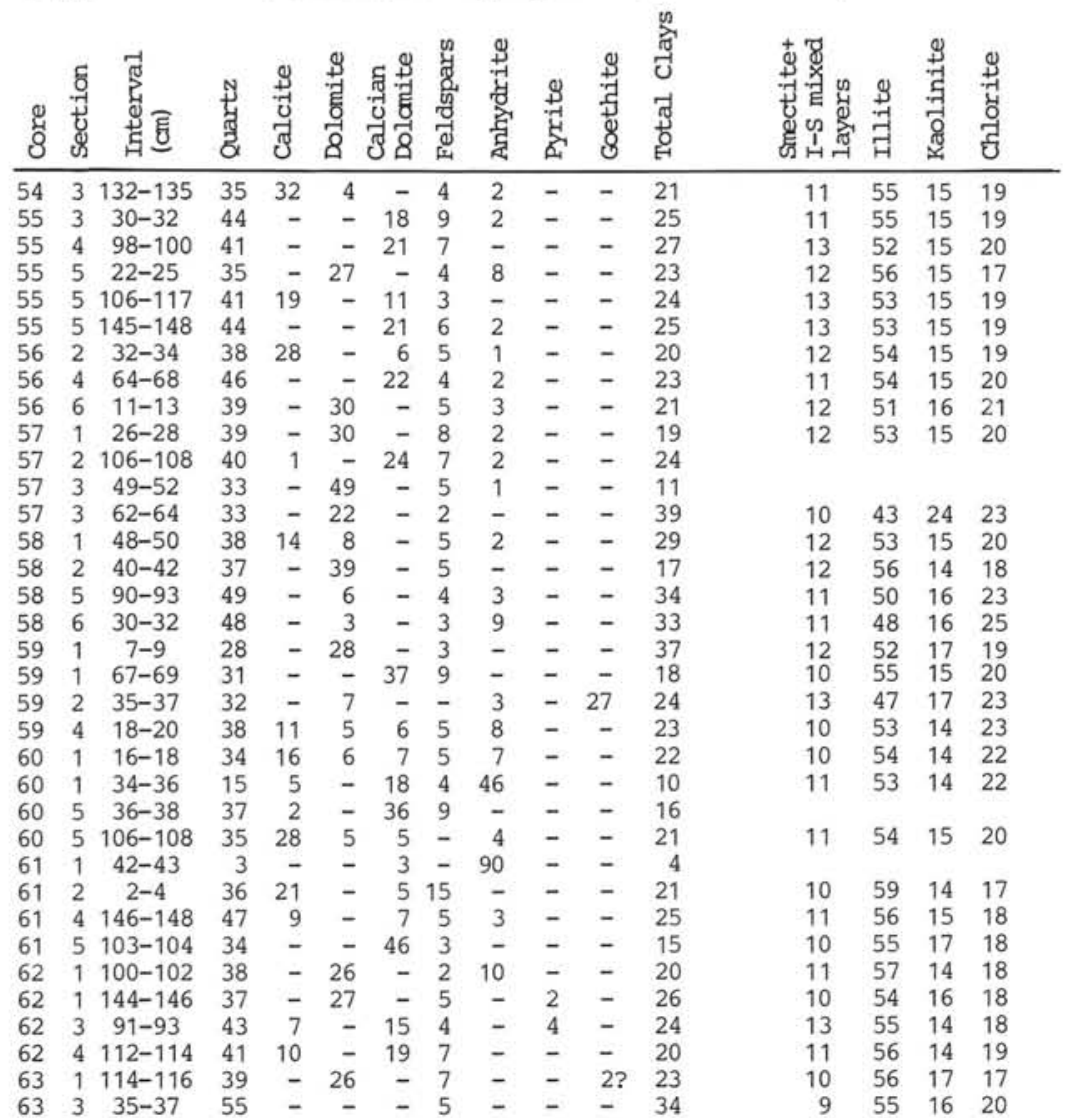

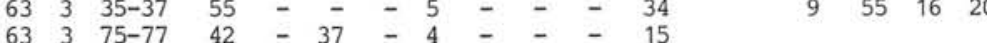

$\begin{array}{lllllrllllll}64 & 1 & 83-85 & 27 & 29 & 4 & 7 & 2 & 9 & 4 & - & 15\end{array}$

$641105-107 \quad 4026-2-45-20$

$64359-6131-51-6-50-11$

$65170-7248-19-4-526 \quad-26 \quad 941522$

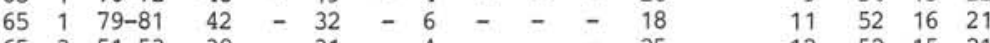

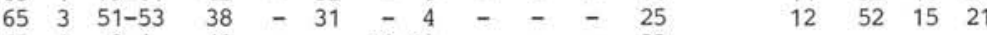

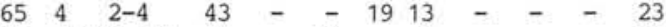

65 4 121-122 $39-39-6-56-14$

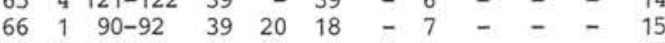

$\begin{array}{llllllllllllll}66 & 3 & 74-76 & 36 & 31 & 4 & 4 & 6 & - & - & - & 17\end{array}$

$\begin{array}{lllllllllll}66 & 3 & 109-112 & 29 & 31 & 5 & 5 & 6 & 5 & - & -\end{array}$

$\begin{array}{lllllllllll}67 & 1 & 107-109 & 30 & 2 & - & 48 & 7 & - & - & 11\end{array}$

$673114-12935-40-4-49$

$\begin{array}{lllllllllll}67 & 4 & 74-75 & 34 & 27 & - & 19 & 4 & - & - & -15\end{array}$

$675132-133406-328-5-514$

$\begin{array}{llllllllllll}67 & 5 & 144-146 & 34 & 23 & 5 & 5 & 9 & - & - & - & 22\end{array}$

$681146-1484513-76-70-25$

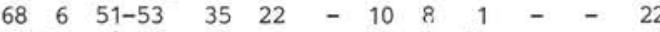

$\begin{array}{llllllrlrlll}68 & \propto C & 8-10 & 20 & - & - & 28 & 3 & 31 & - & - & 16 \\ 69 & 1 & 2-4 & 36 & 27 & - & 9 & 4 & 3 & - & - & 19\end{array}$

$69289-9031-41-7-70$

$692125-1263-5-817-3$

$\begin{array}{llllllllllllll}69 & 4 & 70-72 & 36 & 28 & 7 & 5 & 4 & - & - & - & 18\end{array}$

$\begin{array}{llllllllllll}70 & 2 & 64-66 & 32 & 20 & 7 & 9 & 9 & 2 & - & - & 17\end{array}$

$70377-7933-45-5 \div-54$

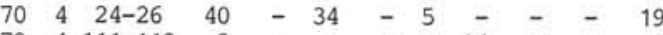

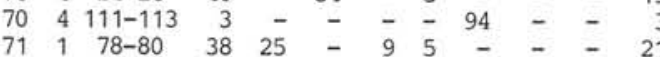

$711128-1303524-77-5=25$

$\begin{array}{llllllllllllll}71 & 2 & 45-47 & 36 & 13 & 8 & 10 & 7 & 1 & - & - & 23\end{array}$

$\begin{array}{lllllllllllll}71 & 2 & 129-131 & 37 & 14 & - & 25 & 7 & - & - & 17\end{array}$

$\begin{array}{lllllllllll}72 & 1 & 54-56 & 37 & 37 & 4 & - & 7 & - & - & -\end{array}$

$\begin{array}{llllllllll}73 & 1 & 17-18 & 36 & 39 & 5 & - & 8 & - & -\end{array} 1$

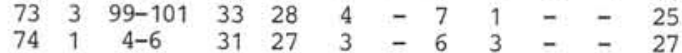

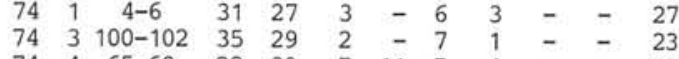

$\begin{array}{lllllllllllll}74 & 4 & 65-69 & 28 & 30 & 7 & 11 & 7 & 4 & - & - & 13\end{array}$

$\begin{array}{llllllll}74 & 5 & 52-54 & 34 & - & 28 & 5 & -\end{array}$

\begin{tabular}{llllllrlllll}
75 & 4 & $23-25$ & 37 & 24 & - & 8 & 6 & - & - & - & 23 \\
75 & 4 & $72-74$ & 41 & 23 & 3 & 3 & 7 & - & - & - & 21 \\
\hline
\end{tabular}

\begin{tabular}{rrrr}
10 & 55 & 13 & 22 \\
9 & 56 & 13 & 22 \\
& & & \\
12 & 52 & 15 & 21 \\
11 & 49 & 18 & 22 \\
& & & \\
12 & 52 & 14 & 22 \\
10 & 50 & 16 & 24 \\
10 & 55 & 14 & 21 \\
11 & 58 & 13 & 18 \\
11 & 53 & 14 & 22 \\
11 & 53 & 15 & 21 \\
12 & 47 & 17 & 24 \\
11 & 52 & 14 & 23 \\
& & & \\
12 & 51 & 15 & 22 \\
& & & \\
11 & 52 & 14 & 23 \\
11 & 53 & 13 & 23 \\
11 & 57 & 11 & 21 \\
11 & 53 & 14 & 22 \\
& & & \\
10 & 56 & 11 & 23 \\
10 & 58 & 10 & 22 \\
9 & 56 & 9 & 23 \\
8 & 57 & 13 & 22 \\
9 & 53 & 14 & 24 \\
8 & 56 & 13 & 23 \\
\hline
\end{tabular}




\begin{tabular}{|c|c|c|c|c|c|c|c|c|c|c|c|c|}
\hline ஜ્પુ & 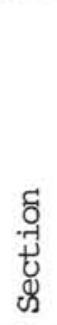 & 莺 & 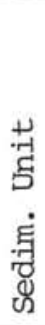 & 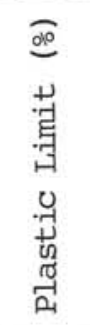 & 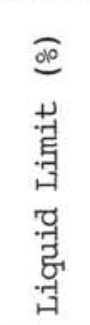 & 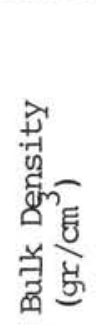 & 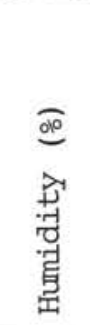 & 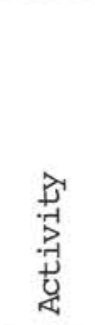 & 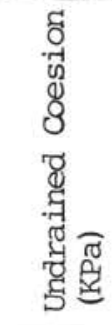 & 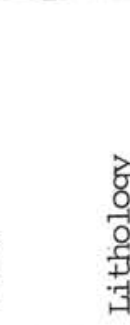 & & 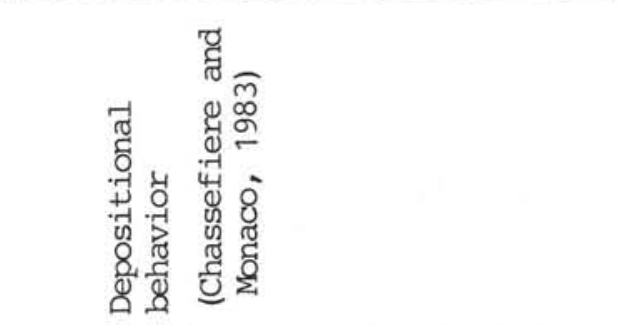 \\
\hline 26 & 11 & $134-142$ & 4 & 23.4 & 48.9 & 2.15 & 20.8 & 0.43 & $>100$ & Clayey & silt & Gulf and bays \\
\hline 28 & 21 & $100-114$ & 4 & 21.7 & 24.0 & 2.27 & 18.3 & 0.53 & " & & $"$ & " " " " \\
\hline 31 & 1 & $81-95$ & 4 & 17.0 & 39.0 & 2.25 & 18.9 & 0.91 & $"$ & $"$ & $"$ & Cont.shelf w.deltaic sedimentation \\
\hline 50 & 2 & $90-103$ & 5 & 23.2 & 50.7 & 2.36 & 14.6 & 0.75 & " & $"$ & $"$ & Gulf and bays \\
\hline 55 & 51 & $106-117$ & 5 & 20.0 & 43.1 & 2.41 & 12.3 & 0.61 & " & " & $"$ & " " " \\
\hline 67 & 31 & $114-129$ & 5 & 17.4 & 40.6 & 2.56 & 9.6 & 0.45 & $"$ & $"$ & $"$ & Cont.shelf w.deltaic sedimentation \\
\hline 74 & 4 & $65-79$ & 5 & 11.8 & 34.0 & 2.70 & 7.8 & 0.02 & " & $"$ & $"$ & Prodeltas or littoral \\
\hline
\end{tabular}

instance, on a continental shelf with deltaic influence or in gulfs and bays (Chassefiere and Monaco, 1983).

These environmental indications, compared to the previously described data on physical sedimentology and mineralogy, suggest that the above method is not applicable in Site 652, where burial and thermal diagenesis are quite strong.

\section{Discussion}

The general character of the pre-Pliocene sequence at Site 652 can be summarized as follows.

1. The whole sequence, including the syn-rift as well as the possible pre-rift interval (bottom Unit 5) belongs to the Messinian, as suggested by the uniformity of sedimentation, of clay mineral composition, and by the constant presence of sulfates down to the lowermost cores. This implies a very high subsidence rate, with more than $500 \mathrm{~m}$ of Messinian sediments deposited essentially under the same conditions.

2 . The pre-rift sequence is mainly arenitic and is more lithified than the overlying one. Extrabasinal clasts include lowgrade metamorphic rocks and a deep water carbonate flysch sequence of Cretaceous to Paleogene age. The same sources apply for the coarse conglomeratic interval (335-344.3 mbsf) separating Unit 4 from Unit 5 (see Sartori et al., this volume, for a description of the conglomerate clasts).

3. Slumps indicating bottom instability are much more frequent in the syn-rift (from 690 mbsf upward) than in the pre-rift sequences. This observation suggests synsedimentary tectonism.

4. Although the sequence is rather monotonously made by fine-grained turbidites, the following superposed intervals can be distinguished from the bottom upward: (a) thin-bedded turbidites with a prominent sand body, coarsening upward and about $5 \mathrm{~m}$ thick, from 721 to about $690 \mathrm{mbsf}$; (b) thin-bedded, rhythmic, silty-shaly turbidites and dark shales more or less disturbed by slumping from some 690 to some $422 \mathrm{mbsf}$; (c) thinbedded, rhythmic, silt-shale and sand-shale couplets with nodular and micronodular anhydrite scattered or aligned in mm-thick "laminae," with some concentration of nodules in massive to inversely graded units, from about 422 to about 344 mbsf; (d) a conglomerate layer from about 344 to $335 \mathrm{mbsf}$; and (e) thinbedded, rhythmic, silt-shale couplets $2-25 \mathrm{~cm}$ thick, lighter than the underlying sequences, with local bioturbation, sulfate clasts, and rare brackish water forms.

5. The couplets found in intervals (c) and (e) may derive from density flows with suspended, mostly fine, detritus. Con- sidering the regularity of bedding, the vertical changes in thickness among couplets could suggest seasonal or some other regular periodicity.

6. The presence of anhydrite rather than gypsum, the latter being by far most common on Messinian land outcrops, can be explained both in terms of dehydration of former gypsum under load and/or of the high thermal gradient observed in the area (Kastens, Mascle, et al., 1987).

7. Sulfate textures in interval (c) are rather ambiguous since they support either deposition of thin sulfate "laminae" or displacive veins parallel to bedding. Under the first assumption, the primary material could have been gypsum crystals precipitating from brines, clastic gypsum, or fragmental particles of anhydrite reworked from sabkha environments. The nodules could also have been displaced (as suggested by some worn-out edges) or have grown in situ. Even in the latter case, however, the embedding lithofacies hardly indicate a sabkha-like environment, so that an origin by burial diagenesis is preferred. Finally, part of the disseminated anhydrite detected by $\mathrm{x}$-ray analysis inside intervals (b) and (c) could derive by diagenesis of pyriterich dark shales, via oxidation to gypsum (later turned to anhydrite) and to iron oxides/hydroxides (the bright red and yellow millimeter-thick laminae observed in many cores).

The studied sequence reflects an environment characterized by high sedimentation and subsidence rate, onsetting during the Messinian inside a half-graben tilted block. Although diluted by the strong fine-grained turbiditic input, the sequence includes prevailing dark shales (interval (b)) overlain by prevailing sulfates (interval (c)). This recalls what is observed everywhere inland around the Tyrrhenian Basin, where the transition from the bituminous Tripoli Formation to the evaporites of the Gessososolfifera Formation occurs. True salt (halite) may be present laterally to the drilled site inside the depocenter of the half-graben. The main difference with the inland exposed sequences is that at Site 652 , the sedimentary facies always record a somewhat deep basin. The conglomerate (interval (d)) may indicate a major tectonic/sedimentary event above which more oxygenated sediments, analogous to those of the "Lago-Mare" of the inland terminal Messinian, are present (interval (e)). We can not exclude, however, that the whole pre-Pliocene sequence only belongs to upper Messinian, as could be indicated by the constantly reverse magnetic signature of the sediments (Channell et al., this volume). In this case, syn-rift tectonism would have started in upper Messinian in the region of Site 652. 


\section{SITE 654}

\section{Location and Structural Setting}

The site is located on the upper Sardinia margin, east of the Baronie Ridge, at a water depth of $2218 \mathrm{~m}$ (Fig. 1). The crustal thickness in this area is about $20 \mathrm{~km}$ (Recq et al., 1984).

Drilling was performed on a tilted block, bounded by eastfacing listric normal faults responsible for basin stretching. More information and details on the site are found in Kastens, Mascle, et al. (1987), from where most data presented below were derived.

\section{Seismic Stratigraphy}

Seismic profiles across the site exhibit a geometry suggestive of pre-, syn-, and post-rift sequences (Fig. 6). Four seismic units (S.U.) are recognized at the site location.

S.U. I. $0-0.3 \mathrm{~s}$ of penetration, interval velocity some $1.6 \mathrm{~km} / \mathrm{s}$. The unit is rather constantly transparent, except for a discontinuous strongly diffractive interval located roughly at the middle. It is bounded to the bottom by a high amplitude reflector.

S.U. II. $0.30-0.42 \mathrm{~s}$ of penetration, interval velocity some 1.6 $\mathrm{km} / \mathrm{s}$. It consists of a series (7-8) of well-layered, closely spaced, horizontally disposed, high amplitude reflectors.

S.U. III. $0.42-0.64 \mathrm{~s}$ of penetration, interval velocity some $2.2 \mathrm{~km} / \mathrm{s}$. The upper portion contains a series of high amplitude reflectors, whereas the lower portion (bottom $0.14 \mathrm{~s}$ ) is almost acoustically reflection-free. A major change in acoustic properties should occur at the boundary between the two portions (at about $420 \mathrm{mbsf}$ using the above interval velocities).

S.U. IV. From $0.64 \mathrm{~s}$ of penetration downward, boundary with the acoustic basement not observed, interval velocity some $4.5 \mathrm{~km} / \mathrm{s}$. The unit is topped by a very high amplitude northnorthwest-dipping reflector. It contains a few, discontinuous and well marked reflectors, parallel to the top one, and grades progressively downward to a reflection-free sequence.

The general arrangement of such units clearly shows that S.U. I is post-rift, S.U. III is syn-rift, and S.U. IV is pre-rift in terms of stretching of the Sardinia margin. S.U. II partly onlaps S.U. III, and partly thickens toward the north-northwest (late syn-rift to early post-rift).

\section{Lithology and Stratigraphy}

At Site $654,478.3 \mathrm{~m}$ of sequence was drilled, and six major sedimentary units were recognized (Fig. 6).

Unit 1. Cores 107-654A-IR to 26R; 0-242.7 mbsf. Dominant nannofossil oozes, with one layer of basalt at 71-73 mbsf; age Pleistocene and Pliocene.

Unit 2. Cores 107-654A-27R to $36 \mathrm{R}-1,110 \mathrm{~cm} ; 242.7-312.6$ mbsf, thickness $69.9 \mathrm{~m}$. Interbedded gypsum layers and carbonate layers; age Messinian.

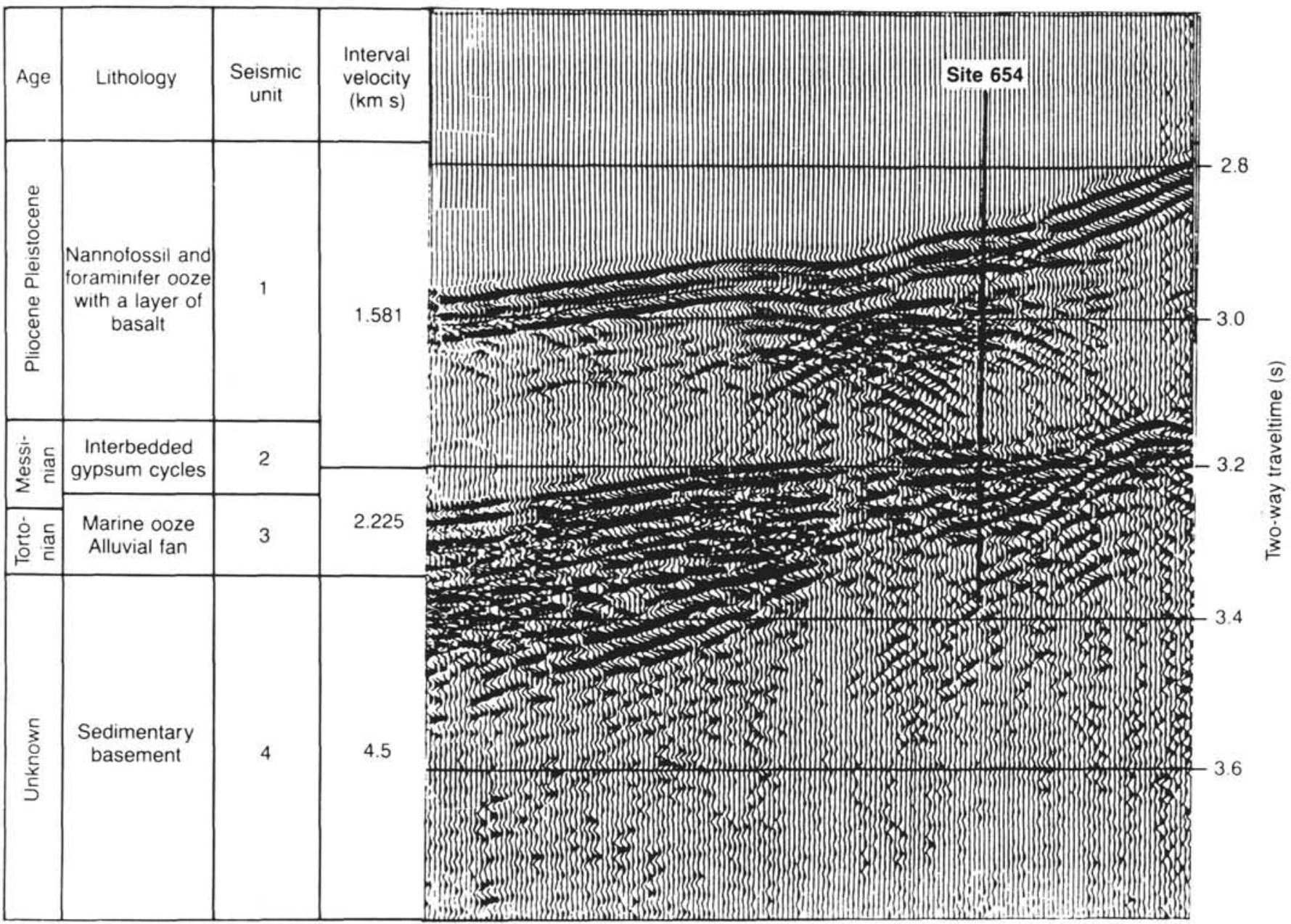

Figure 6. Seismic profile and schematic interpretation of the sequences drilled at Site 654 (from Kastens, Mascle, et al., 1987). 
Unit 3. Cores $107-654 \mathrm{~A}-36 \mathrm{R}-1,110 \mathrm{~cm}$, to $40 \mathrm{R}-1,7.5 \mathrm{~cm}$; 312.6-348.9 mbsf, thickness $36.3 \mathrm{~m}$. Dark, laminated, organicrich pelites displaying debris flow, convolute lamination, and microfaults; age Messinian (lower and middle?).

Unit 4. Cores $107-654 \mathrm{~A}-40 \mathrm{R}-1,7.5 \mathrm{~cm}$, to $45 \mathrm{R}-5,145 \mathrm{~cm}$; 348.9-403.9 mbsf, thickness $55.0 \mathrm{~m}$. Dominant nannofossil oozes; age lowermost Messinian to uppermost Tortonian.

Unit 5. Cores 107-654A-45R-5, $145 \mathrm{~cm}$, to 46R; 403.9-415.7 mbsf, thickness $11.8 \mathrm{~m}$. Polymict greenish sandstone bearing large benthic foraminifers (Operculina type) and thick-walled Ostreyd shells; age not determined (upper Tortonian?).

Unit 6. Cores 107-654A-46R to 52R; 415.7-478.3 mbsf, thickness (minimum) $62.6 \mathrm{~m}$. Reddish gravel-bearing mudstones and conglomerates. Clasts represent a Sardinia-type Hercynian basement with possibly late Paleozoic-early Triassic carbonate rocks (Sartori et al., this volume); age not determined.

\section{Seismic and Lithologic Correlations}

S.U. I corresponds to Unit 1; that is the post-rift sequences are nannofossil oozes (with a basaltic layer) of Pliocene and Pleistocene age. S.U. II corresponds to Unit 2; that is interbedded gypsum and carbonate intervals of Messinian age are partly post-rift and partly syn-rift. S.U. III corresponds to Units 3, 4, 5 , and 6 , documenting an almost complete transgressive syn-rift sequence that develops from at least upper Tortonian to lower Messinian passing from subaerial conglomerates (6) to arenites (5) to nannofossil oozes (4) until the onset of Messinian salinity crisis (dark, organic-rich pelites of Unit 3). Pre-rift sequences (S.U. IV) have not been reached but their lithotypes should be recorded in the conglomerate clasts and in the sandstone grains.

\section{Physical Sedimentology and Facies Analysis (Fig. 7)}

The basal conglomerate (Unit 6) looks like the basal deposits of a classic sedimentary cycle. As it is framed by finer red beds, the obvious interpretation is that of a continental deposit (alluvial fan?, coarse-grained delta?, fan delta?).

The conglomerate is overlain by a fining-upward sequence, starting with bioturbated, fossiliferous fine sands (Unit 5) which grade upward into bioturbated, light muds (Unit 4). This sequence marks a deepening interface and continues the apparent transgressive trend suggested by the basal conglomerate. The sand should have been deposited in a nearshore-deltaic environment, the mud in an offshore area below wave base (prodelta, shelf).

An interval of dolomitic dark shales follows, marking a phase of anoxia (Unit 3). Regular, flat bedding, and thin alternations of fine sand, silt, and shale characterize this interval. Silt and sand beds are graded, and the overall aspect is that of "varved" sediments settling on a quiet bottom. A basinal setting is suggested with stagnant stratified water, possibly protected by topographic barriers. There is no indication of depth and, in contrast with the dark shale section of Site 652, evidence of slope instability is quite scarce. Some thin layers of deformed fibrous gypsum can indicate either secondary emplacement of remobilized sulfate (displacive veins) or disturbance of primary gypsum. Disturbed shales can be noticed at various levels. The geometry of deformation does not suggest slumping but shocks or, in some cases, bioturbation. The thickness of shales is about $34 \mathrm{~m}$.

A gypsum section, comprised mainly of laminated, "balatino"-like gypsum in small crystals follows (Unit 2). Layers about a millimeter to a centimeter thick of gypsum are also frequent, together with beds of white saccharoidal gypsum (alabastrino). The gypsiferous sands alternate with thin shale or "alabastrino" respectively. In the former case, small density flows are suggested as the main mechanism of emplacement. In the latter type of couplet, surfaces of erosion or dissolution sepa-

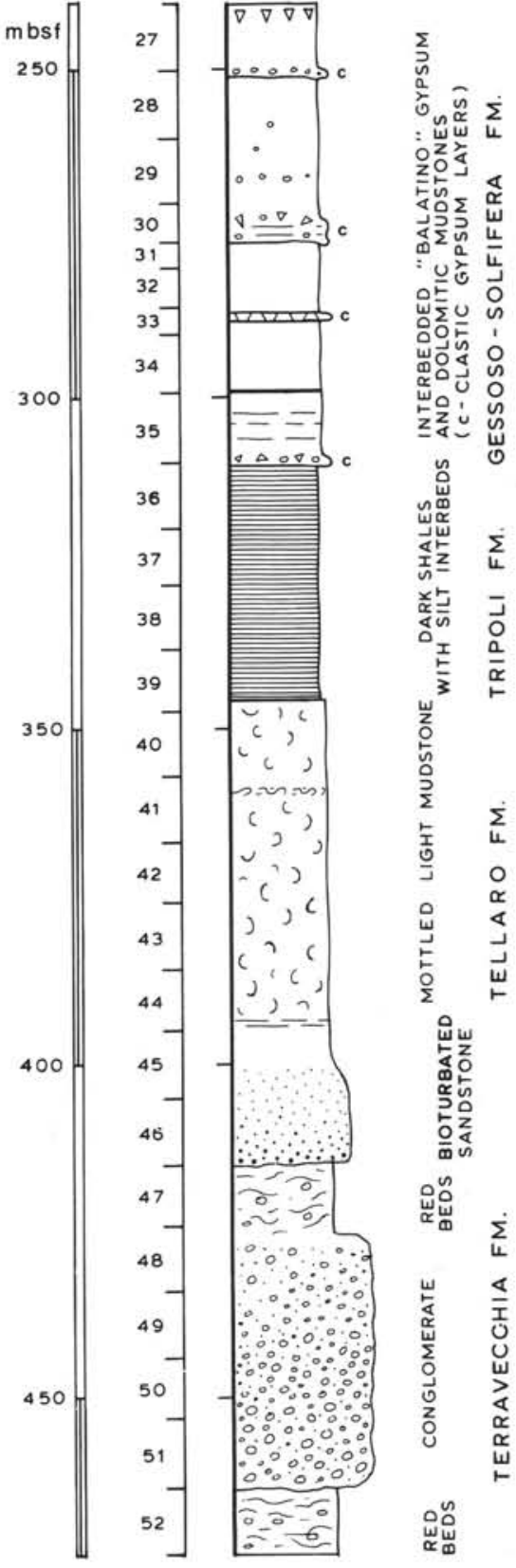

Figure 7. Schematic pre-Pliocene sequence in Site 654, with the possible equivalences to formations cropping out in Sicily.

rate the two members. This is a possible indication of subaerial exposure or, at least, of mechanical reworking in shallow water.

The "alabastrino" records, in this case, the growth of nodules and micronodules confined in thin layers of host material (lateral growth or coalescence). The gypsarenite beds could represent resuspension of gypsum sand by storm events in shallow water and redeposition below storm wave base. A shallow lagoon (shallow refers only to water depth) with associated intratidal to supratidal flats is a reasonable environment of formation for this facies. Isolated nodules or crystals of selenite, reported from many cores, find a plausible place in this setting. A layer with gypsum fragments embedded in a gypsum-clay matrix at the base of the interval marks an episode of debris flow involving gypsum originally emplaced in a more marginal position (subaerial flow?). 


\section{Arenite Composition}

Six samples from Cores $44 \mathrm{R}$ to $46 \mathrm{R}$ were analyzed. A particular feature of these arenites is that sand grains display alteration and replacement fabric by glauconite.

The terrigenous siliciclastic component (NCE) is made up of a variety of low-grade metamorphic rock fragments, small amounts of quartz, rare feldspars, micas, and heavy minerals (Table 2). In contrast to samples from Site 652, terrigenous carbonate lithoclasts are absent but a large quantity of intrabasinal carbonate grains are present. They consist of benthonic foraminifers mostly of the genus Operculina, present both as large unbroken individuals and as sand grains of broken tests (Fig. $4 C$ ). Fine-grained metamorphic rock fragments constitute the principal substrate for glauconite formation and all transitions between unaltered to deeply altered (but still recognizable) grains can be observed (Fig. 4D). The substrate of glauconitization can also be micas, internal moulds, skeletal remains, quartz, and feldspars. No detailed analyses have been performed in order to characterize the different types of glauconite present (Hughes and Whitehead, 1987). Only "glauconite replacement on skeletal clasts" and "glauconite as internal moulds" have been assigned to the intrabasinal carbonate component. Although glauconite replacement on low-grade metamorphic rocks formed in intrabasinal conditions, glauconitized lithics were assigned to the "L group" because the terrigenous nature of these grains was always detectable. On the contrary, glauconite internal moulds and replaced skeletal grains originated by the alteration of intrabasinal particles were assigned to the noncarbonate intrabasinal group (NCI).

A significative amount of microspar in an interstitial position is thought to be recrystallized carbonate matrix and thus assigned to this class.

Figure 8 represents a first-level classification of the principal types of grains making up the framework component of these arenites group. According to Zuffa (1980), they can be classified as "hybrid arenites" l.s., since they are composed of intrabasinal (i.e., coeval) and extrabasinal (i. e., noncoeval) grains. In the QFL classification of the siliciclastic terrigenous component (NCE), samples plot in the field of pure litharenite (Fig. 8B).
From the data illustrated above we can outline some paleogeographic constraints of the source/basin system and related evolution through time:

1. Low-grade metamorphic crystalline rocks characterized the hinterland source area;

2. Glauconitization of terrigenous siliciclastic substratum, according to Odin (1985), would imply a marine environment between 50 and $500 \mathrm{~m}$ depth with conditions of a lack of deposition; and

3. The glauconitization process took place together with production of shallow-marine carbonate factories (Foramol) (Lees and Buller, 1972; Kamp and Nelson, 1987).

\section{X-ray Diffraction Analysis}

Samples from the Cores $38 \mathrm{R}-50 \mathrm{R}$ were analyzed. Their bulk and clay mineralogy can be used to draw sharp boundaries among the different lithologic units (Table 5).

Unit 3 in Cores $38 \mathrm{R}$ and $39 \mathrm{R}$ shows a very high dolomite content $(50 \%-70 \%)$ and moderate quartz amounts $(10 \%-20 \%)$. It is characterized by the presence of pyrite $(2 \%-6 \%)$ and gypsum (up to $2 \%$ ). Clay minerals include the highest amounts of smectite $(30 \%-50 \%)$, relevant illite percentages $(40 \%-50 \%)$, and subordinate kaolinite and chlorite.

The beginning of the underlying Unit 4 (nannofossil oozes) is emphasized by a remarkably high calcite content $(40 \%-65 \%)$ and by a sudden decrease of dolomite to $1 \%-6 \%$. Smectite decreases gradually and in the lower part of the unit (Cores 43R and 44R) illite-smectite mixed-layers become dominant. This is paralleled by the presence of Ca-dolomite in amounts up to $10 \%-20 \%$. Illite gradually increases downward and chlorite content is higher than the kaolinite content.

The transition to Unit 5 (glauconitic sandstones) is indicated by a progressive decrease in calcite, paralleled by an increase in quartz (up to $40 \%$ ). Kaolinite disappears and expandable clay minerals become scarce, while illite $(60 \%-70 \%)$ and chlorite (up to more than $20 \%$ ) strongly increase.

The oldest analyzed sediments (the clay of Core 47R and the matrix of conglomerates in Core 50R) show a very low calcite content (about 5\%) with remarkable quartz amounts (more than

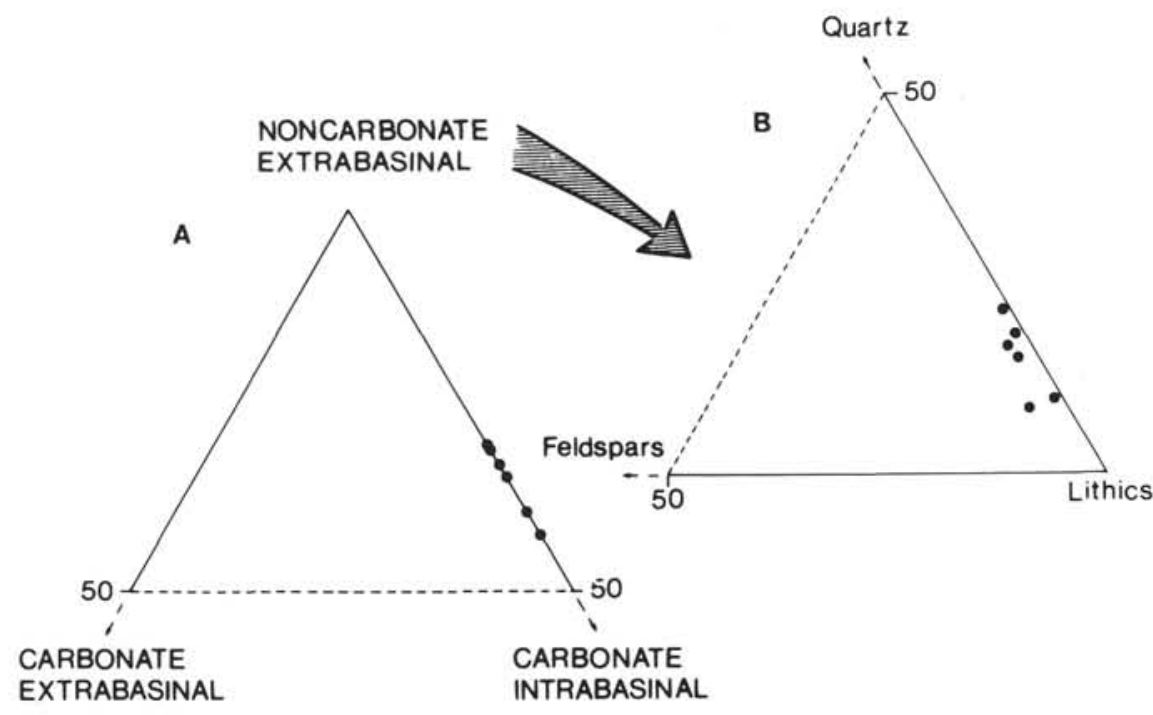

Figure 8. Framework composition of arenites from Site 654. A. Composition of main types of grains (first-level classification). B. Composition of the noncarbonate extrabasinal component (second-level classification). 
Table 5. X-ray mineralogy of Site 654.

\begin{tabular}{|c|c|c|c|c|c|c|c|c|c|c|c|c|c|c|c|}
\hline \multicolumn{4}{|c|}{ Sample } & \multicolumn{8}{|c|}{ Mineral percentages (powders) } & \multicolumn{4}{|c|}{ Clay Minerals (100\%) } \\
\hline 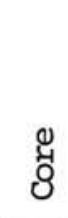 & 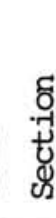 & 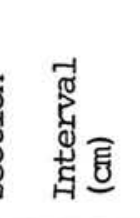 & $\begin{array}{l}\stackrel{N}{4} \\
\text { 范 }\end{array}$ & 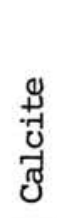 & $\begin{array}{l}9 \\
\stackrel{-1}{0} \\
\text { 어 }\end{array}$ & 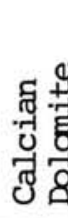 & 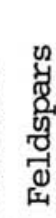 & 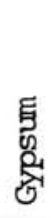 & 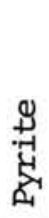 & 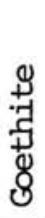 & 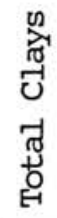 & 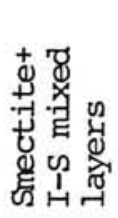 & 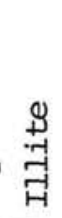 & 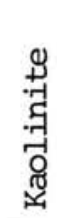 & 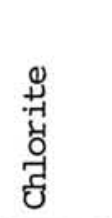 \\
\hline 38 & 2 & $7-12$ & & & & & & & & & & 47 & 37 & 8 & 8 \\
\hline 38 & 2 & $89-93$ & 13 & - & 70 & - & 3 & 1 & 2 & - & 11 & 40 & 41 & 9 & 10 \\
\hline $38 \mathrm{c}$ & $\mathrm{CC}$ & $8-10$ & 13 & - & 65 & - & 4 & - & 4 & - & 12 & 36 & 41 & 12 & 11 \\
\hline $38 c$ & CC & $12-15$ & 16 & - & 56 & - & 8 & 1 & 4 & - & 15 & 41 & 40 & 9 & 10 \\
\hline 39 & 1 & $39-42$ & 16 & - & 64 & - & 3 & - & 6 & - & 10 & 42 & 41 & 8 & 9 \\
\hline 39 & 1 & $60-62$ & 14 & - & 67 & - & 3 & 2 & 2 & - & 12 & 44 & 38 & 9 & 9 \\
\hline 39 & 2 & $144-150$ & 25 & - & 33 & - & 10 & tr & 4 & - & 28 & 26 & 49 & 10 & 15 \\
\hline 39 & 3 & 3-4 & 21 & - & 38 & - & 12 & tr & 4 & - & 25 & 52 & 31 & 6 & 11 \\
\hline 39 & $\mathrm{CC}$ & $3-5$ & 22 & - & 55 & - & 5 & - & 3 & - & 15 & 29 & 49 & 11 & 11 \\
\hline 40 & 1 & $137-139$ & 19 & 14 & 50 & - & 5 & 1 & - & - & 11 & 30 & 46 & 11 & 13 \\
\hline 40 & 2 & $60-63$ & 23 & 55 & 5 & - & 4 & tr & - & - & 13 & 15 & 53 & 15 & 17 \\
\hline 40 & 5 & $65-67$ & 30 & 43 & 2 & - & 6 & $\operatorname{tr}$ & - & - & 19 & 18 & 50 & 15 & 17 \\
\hline 41 & 1 & $70-73$ & 22 & 59 & 2 & - & 5 & tr & - & - & 12 & 23 & 48 & 14 & 15 \\
\hline 41 & 2 & $145-150$ & 21 & 62 & 2 & - & 3 & - & - & - & 12 & 26 & 46 & 14 & 14 \\
\hline 41 & 4 & $69-72$ & 22 & 50 & 2 & - & 9 & tr & 2 & - & 15 & 27 & 46 & 11 & 16 \\
\hline 41 & 4 & $101-103$ & 30 & 42 & 2 & - & 6 & - & - & - & 18 & 23 & 51 & 11 & 15 \\
\hline 42 & 1 & $69-72$ & 18 & 65 & 1 & - & 4 & tr & - & - & 11 & 14 & 54 & 15 & 17 \\
\hline 42 & 5 & $97-99$ & 17 & 66 & 2 & - & 2 & tr & - & - & 13 & 12 & 52 & 15 & 21 \\
\hline 42 & 6 & $21-24$ & 14 & 67 & 2 & - & 6 & - & - & - & 11 & 7 & 55 & 15 & 23 \\
\hline 43 & 2 & $107-110$ & 15 & 61 & 1 & - & 8 & - & - & - & 13 & 6 & 61 & 13 & 20 \\
\hline 43 & 3 & $130-132$ & 15 & 66 & 2 & - & 4 & - & - & - & 12 & 6 & 62 & 11 & 21 \\
\hline 43 & 4 & $145-150$ & 13 & 65 & 2 & - & 2 & - & - & - & 18 & 5 & 61 & 13 & 21 \\
\hline 43 & 5 & $0-5$ & 15 & 66 & 1 & - & 2 & - & - & - & 14 & 6 & 60 & 13 & 21 \\
\hline 43 & 5 & $130-133$ & 17 & 56 & 2 & - & 6 & - & - & - & 17 & 7 & 63 & 10 & 20 \\
\hline 44 & 1 & $145-147$ & 16 & 61 & 2 & - & 4 & tr & - & - & 16 & 5 & 62 & 12 & 21 \\
\hline 44 & 2 & $109-112$ & 17 & 43 & 6 & 8 & 4 & tr & - & - & 19 & 5 & 68 & 8 & 19 \\
\hline 44 & 6 & $2-4$ & 16 & 38 & - & 22 & 6 & - & - & - & 16 & 7 & 65 & 8 & 20 \\
\hline 44 & 6 & $96-99$ & 19 & 46 & - & 12 & 3 & $\operatorname{tr}$ & - & - & 18 & 7 & 67 & 7 & 19 \\
\hline 45 & 1 & $80-82$ & 19 & 44 & - & 7 & 5 & - & - & - & 24 & 8 & 68 & - & 24 \\
\hline 45 & 5 & $126-131$ & 24 & 34 & 1 & - & 9 & - & - & - & 29 & 5 & 75 & - & 20 \\
\hline 45 & 6 & $14-20$ & 28 & 42 & tr & - & 7 & tr & - & - & 21 & 5 & 74 & - & 21 \\
\hline 47 & 1 & $2-4$ & 40 & 8 & - & - & 11 & $\operatorname{tr}$ & - & - & 38 & - & 82 & - & 18 \\
\hline 47 & 1 & $84-86$ & 33 & 8 & - & - & 13 & - & - & - & 43 & - & 84 & - & 16 \\
\hline 50 & 1 & $93-95$ & 32 & 6 & - & - & 13 & - & - & - & 45 & - & 84 & - & 16 \\
\hline 50 & 1 & $117-119$ & 26 & 23 & - & - & 13 & - & - & - & 38 & - & 83 & - & 17 \\
\hline
\end{tabular}

$30 \%$ ). Clay minerals of Unit 6 are totally different from those of the above sediments. Only the most stable phases are present (average $85 \%$ of illite and $15 \%$ of chlorite).

\section{Physical Properties of Sediments}

In contrast to Site 652 , Site 654 shows a lesser diagenetic overprint, so that indications from Atterberg limits better match with sedimentologic inferences. The examined samples (from Units 2, 3, and 4) are inactive or weakly active silty clays (Skempton, 1953) which indicate shallow gulfs and bays or a continental shelf with deltaic sedimentation for Units 2 and 3 (Table 6). The sample at $\mathbf{3 6 2 . 2 5}$ mbsf in Unit 4 (nannofossil oozes) is an active silty clay whose properties recall those of present-day central Mediterranean bathyal deposits (Keller and Lambert, 1972). The sample from bottom Unit 4 (383.05 mbsf), toward the passage to Unit 5, again indicates depositional behavior in gulfs and bays.

\section{Discussion}

The pre-Pliocene sequence recorded in the site shows the onset and development of a typical sedimentary cycle. A continental substrate represented by pre-Jurassic rocks of the Sardinia basement and of Tuscany (Sartori et al., this volume) is transgressed by Ostreid- and Operculinid-bearing glauconitic sandstones passing upward to deeper water nannofossil oozes. The overlying carbon-rich dark shales and gypsum layers record the onset of the Mediterranean salinity crisis by progressive isolation and dramatic shallowing of the water depth.

A critical point for any discussion regards the continuity in time for deposition of the sequence, mainly as concerns the age of the transgressive basal sandstones. There are two possibilities:

1. The glauconitic sandstones are lower Miocene in age. This may be suggested by the fact that, regionally, facies very 
Table 6. Physical properties at Site 654.

\begin{tabular}{|c|c|c|c|c|c|c|c|c|c|c|}
\hline 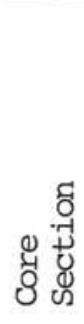 & 莺 & 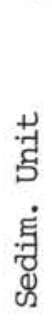 & 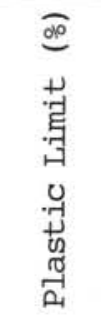 & 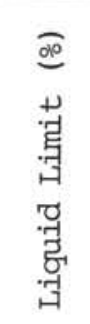 & 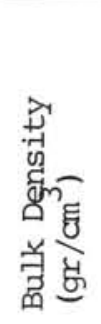 & 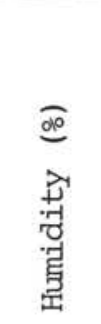 & 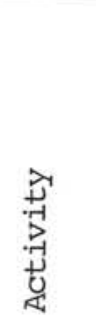 & 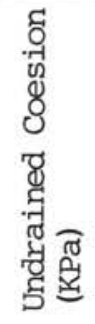 & 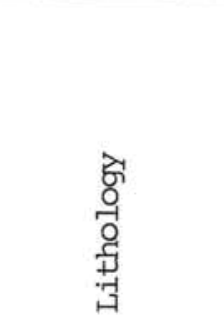 & 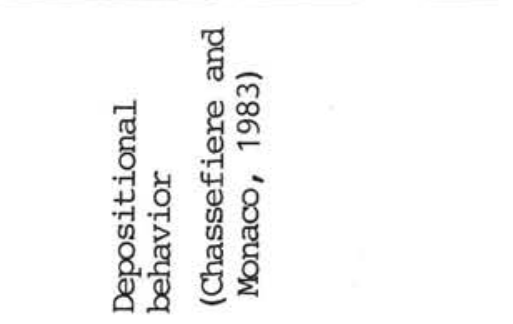 \\
\hline 351 & $60-74$ & 2 & 25.6 & 53.2 & 2.20 & 21.3 & 0.44 & $>170$ & Silty clay & Gulfs and bays \\
\hline 392 & $144-150$ & 3 & 19.4 & 50.5 & 2.09 & 31.0 & 0.53 & $"$ & Clayey silt & \\
\hline 413 & $145-150$ & 4 & 31.3 & 62.1 & 2.16 & 26.6 & 0.57 & $"$ & Silty clay & Evoluted seds., deep margins \\
\hline 434 & $145-150$ & 4 & 22.9 & 49.5 & 2.24 & 26.0 & 0.39 & $"$ & " " & Gulfs and bays \\
\hline 455 & $126-131$ & 4 & 9.3 & 31.6 & 2.29 & 15.3 & 0.23 & $"$ & Clayey silt & Prodeltas or littoral \\
\hline
\end{tabular}

similar to that of Site 654 are of Burdigalian age. In Corsica, for instance, glauconitic sandstones of that age and containing Ostreids and Operculinids are directly transgressive on the granitic basement of the island (Orszag-Sperber and Pilot, 1976). Moreover, Operculinids are not known in post-middle Miocene sediments of the Mediterranean Basin, and this is generally attributed to the severe worldwide climatic deterioration that occurred between Langhian and Serravallian and is also recorded in Mediterranean sediments (Zachariasse and Spaak, 1983; Vergnaud-Grazzini, 1985).

2. The glauconitic sandstones are upper Tortonian in age. The bottommost datable sediments in Core 45R contain planktonic foraminifers of uppermost Tortonian age and they are intermixed, with a gradual passage and without apparent hiatus, to the upper interval of glauconitic sandstones. The recovery in these key cores is complete. Therefore, we prefer to interpret the age of sandstones as upper Tortonian also on the basis of the following considerations:

a. Operculinids are facies fossils with only local stratigraphic value (Adams et al., 1983). Ostreids and glauconite are well known on land as components of upper Tortonian transgressive sands.

b. The finding of Operculinids in Site 654 recalls the finding of Miogypsinids in the upper Tortonian-Messinian calcarenites of Minorca (Bourrouilh, 1973). Also Miogypsinids are unknown elsewhere after lower-middle Miocene, and these findings pose the alternative of reworking vs. autochthony of the deposits. We can not exclude completely reworking of Operculinids in Site 654 , but it should have been not severe, since the very thin and delicate Operculina shells, often up to $1 \mathrm{~cm}$ in length, are not broken and gently imbricated by bottom currents.

c. In Corsica and in the Corsica Basin (Sartori et al., 1987), the Burdigalian cycle is separated from the Tortonian one by a disconformity and by a hiatus encompassing part of middle Miocene. This is not observed in Site 654 .

\section{CORRELATIONS AND CONCLUSIONS}

In both Sites 652 and 654 evaporites follow shales in the same vertical order. Evaporites are represented by sulfates only: gypsum in Site 654, anhydrite in Site 652. The mineralogical difference can be explained by a different diagenetic history, with deeper burial and higher thermal gradients in Site 652 .

In Site 654 red conglomerates and sandstones are overlain by open marine sediments of upper Tortonian-early Messinian age, by dark shales and by gypsum layers whose facies ("balatino" to "alabastrino") suggest a marginal, shallow-water to suprati- dal setting. The sequence recalls almost exactly the one recorded in Sicily (Fig. 7), with the "postorogenic" coarse detrital Terravecchia Formation overlain by the deep marine Tellaro Formation, by the bituminous Tripoli Formation, and by the "Gessoso-solfifera" Formation or "Gessi inferiori" of Decima and Wezel (1973). In addition to the Sicilian record, similar sequences are found in the Apenninic foredeep and (excluding evaporites) in the "postorogenic" sequences of the coastal chain of Calabria (Di Nocera et al., 1974) and around other segments of the Tyrrhenian margin of Apennines (Fig. 9). Site 654 is located at the margin of a thick evaporitic Messinian basin located in the Cornaglia Terrace (Fabbri and Curzi, 1979; Moussat, 1983) and bounded to the east by the R. Selli Line. Its sequence is a classical example of the so-called "Sahelian cycle" (Ruggieri, 1958; Ricci Lucchi, 1986) recognized in mainland Italy and Sicily.

At Site 652 , on the other hand, the shaly section is much thicker overall, the individual beds are also thicker, and there are indications of a deeper and strongly subsiding environment. Moreover, since the whole drilled sequence belongs to the Messinian, we do not know its base and therefore whether a preeuxinic condition existed before. The drilled section could correspond to the Tripoli Formation and to part of the "Gessososolfifera" Formation although in nonevaporitic facies. In fact, at Site 652 the evaporites are less represented, while the overall section is thicker, and they are probably resedimented by gravity flows and probably partly diagenetic in origin. In principle, they could represent either the same episode as the evaporitic interval in Site 654 or a later episode of remobilization. The postevaporitic sequence in Site 652, with the conglomerate at its base, may be the possible equivalent of the "Lago-Mare" deposits. Alternatively, the whole drilled syn-rift sequence may be of upper Messinian age. The depositional setting does not seem to vary much during the Messinian: a strongly subsiding, basinal condition seems to have persisted in the area of Site 652 . No evidence of sabkha or intertidal facies is observed (the anhydrite nodules could have been displaced), but a rather monotonous repetition of thin-bedded, fine-grained turbidites laid down on an anoxic (beneath the conglomerate) to slightly oxygenated (above the conglomerate) bottom.

While the sediments of Site 652 recall those found in other Messinian turbidite basins, such as the Laga Formation of the Apenninic foredeep (Fig. 9), the regular rhythmicity and the presumable onset of tectonic subsidence are better correlated to the sequences found in more or less coeval grabens of the Tyrrhenian sector of Tuscany. Here the fillings are more continental/transitional in character, and conglomerates sometimes occur within the deposits (Lazzarotto, 1967). 


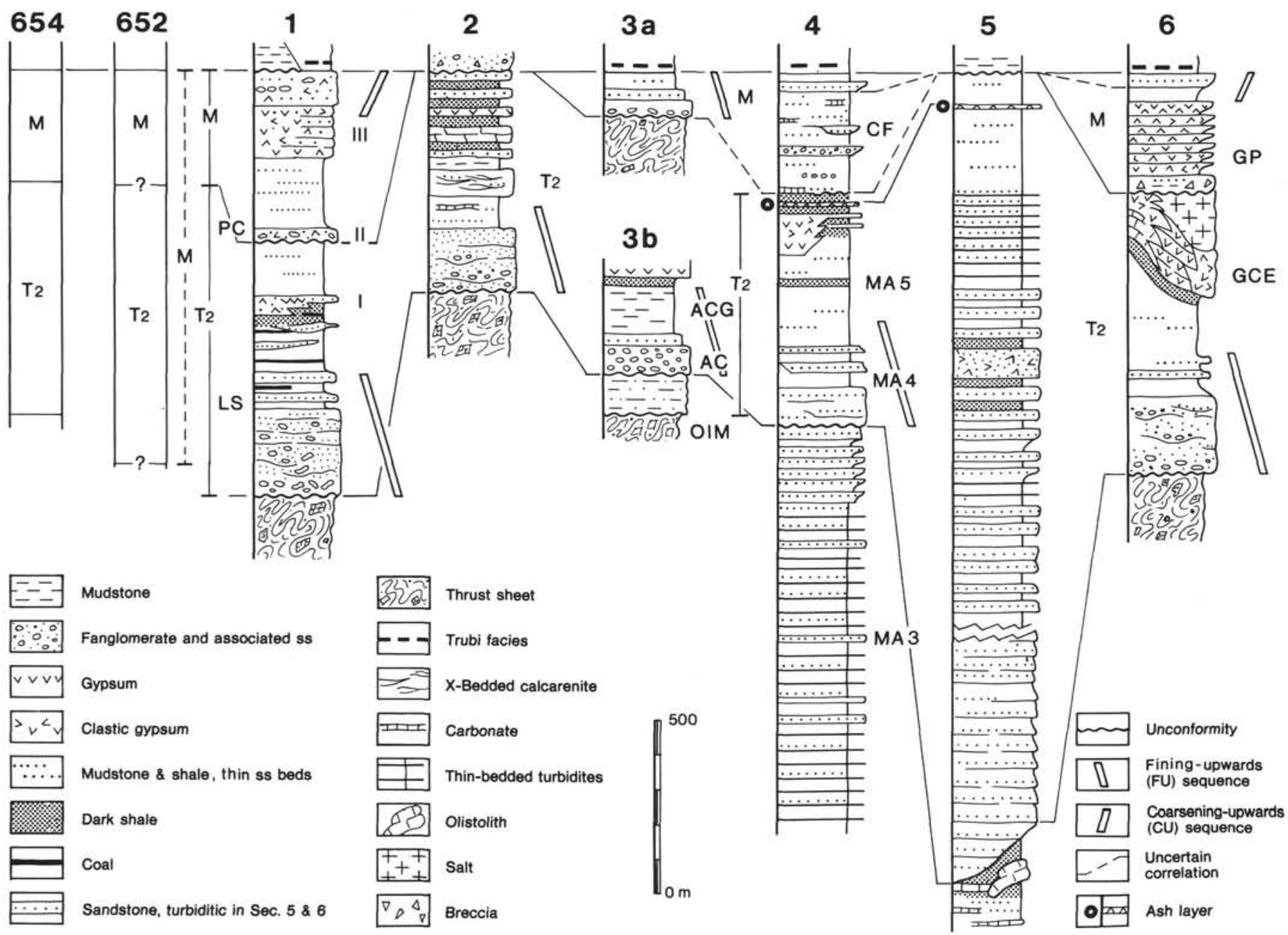

Figure 9. Main reference sections on land arranged in geographical order from north to south and from internal to external portion of Apennines/ Calabria/Sicily orogen. 1. Tuscany (Apennines chain, Tyrrhenian domain, after Lazzarotto and Mazzanti, 1978). 2. Calabria (chain, Tyrrhenian domain, after Perrone et al., 1973). 3. Northern Apennines, thrust top (satellite basins); 3A, West Emilia, after Iaccarino and Papani (1979); 3B, Marecchia Valley, after Ruggieri (1970). 4. Northern Apennines, foredeep, Adriatic domain, after Cremonini and Farabegoli (1977) and Ricci Lucchi (1973). 5. Central Apennines, Marche-Abruzzi foredeep, Adriatic domain, after Girotti and Parotto (1969) and Ricci Lucchi (1973). 6. Sicily, foredeep, after Decima and Wezel (1973) and Nesteroff (1973). Section location is schematically reported in the index map on the upper right side of Figure 1.

\section{EXPLANATORY NOTES:}

T2 and M, main depositional sequences after Ricci Lucchi (1986); 1Q, late Quaternary; mP, middle Pliocene; eP, early Pliocene; OIM, Oligocene-Miocene (Epi-ligurian sequences).

Lithostratigraphic Units: PC, Pycnodonta clays; III, II, I, gypsum horizons; LS, lacustrine sequence; CF, Colombacci Formation; ACG, "Sahelian" clays; AC, Acquaviva Conglomerate; VU, ash layer; MA5, MA4, MA3, upper members of Marnoso-Arenacea Formation (turbidite wedge); LA, Laga Formation (turbidite wedge); GP, Gessi di Pasquasia (= "Upper Gypsum"); GCE, Gessi di Cattolica (= "Lower Gypsum").

Cycles: T2 is the "Sahelian cycle" of late Tortonian-early Messinian age, after Ruggieri (1958); it is well developed east of the Tyrrhenian Sea, and quite correlatable with the fining-upward sequence of ODP Site 654. This period marks the near closure of a northern segment of the Apenninic foredeep (MA4, MA5) and the sudden opening of a new, southern segment (Laga Formation), with an impressive subsidence and initially starved conditions. T2 is unconformable in all outcropping sections, including the all marine ones; its base corresponds to a remarkable phase of submarine erosion (see reference Section 4). In thrust-top sections (3), postdepositional displacement has occurred. Sequence M is limited to the upper Messinian (post-evaporite). Its base, reflecting an "intra-Messinian event" (regarded as tectonic by Decima and Wezel, 1973), can be locally subdued or masked, whereby the Messinian has traditionally been regarded as a continuous section, especially in peninsular Italy. Pycnodonta clays (Section 1) and part of the Colombacci Formation (Section 4) are particularly reminiscent of the rhythmic, clastic/sulfatic sequence of Site 652. The Laga Flysch (Section 5) can also be quoted as a partial analogue, although thick sand beds are much more developed in it. Moreover, the Laga sequence is here tentatively correlated with the T2 cycle, on the base of an ash layer occurring in Romagna between the gypsum and the Colombacci Formation, i.e., the proper $\mathrm{M}$ cycle. In Tuscany, only affected by vertical movements during the Pliocene and Quaternary, both T2 and M sequences are post-orogenic in the current meaning. In outer Apennines, by contrast, they follow important deformational events but they precede the most important (Pliocene) compressional phases leading to the emergence of the chain. 


\section{REFERENCES}

Adams, C. G., Gentry, A. W., and Whybrow, P. J., 1983. Dating the terminal tethyan event. In Meulenkamp J. (Ed.), Reconstruction of marine paleoenvironments. Utrecht Micropaleontol. Bull., 30:273-298.

Aloisi, J. C. and Monaco, A., 1980. Etudes des structures sédimentaires dans les milieux deltaiques (Rhõne). C.R. Acad. Sci. Paris, 290:59162.

Biscaye, P. E., 1965. Mineralogy and Sedimentation of Recent Deep-Sea Clay in the Atlantic Ocean and Adjacent Seas and Oceans. Geol. Soc. Am. Bull., 76:803-832.

Bourrouilh, R., 1973. Stratigraphie, sedimentologie et tectonique de l'ile de Minorque et du Nord-Est de Majorque (Baleares). (Thése Doct. Sci.). Université Paris VI.

Chassefiere, B., and Monaco, A., 1983. On the use of Atterberg limits on marine soils. Mar. Geotechnol., 5:153-179.

Cook, H. E., Johnson, P. D., Matti, J. C., and Zemmels, J., 1975. Methods of sample preparation and x-ray diffraction data analysis, $\mathrm{x}$-ray mineralogy laboratory, Deep Sea Drilling Project, University of California, Riverside. In Hayes, D.E., Frakes, L.A., et al., Init. Repts. DSDP, 28: Washington (U.S. Govt. Printing Office), 9991007.

Cremonini, G., and Farabegoli, E., 1977. Litostratigrafia della Formazione a Colombacci in Romagna. G. Geol., 42:61-82.

Decima, A., and Wezel, F. C., 1973. Late Miocene evaporites of the central Sicilian Basin. In Ryan, W.B.F., Hsü, K. J., et al., Init. Repts. DSDP, 13: Washington (U.S. Govt. Printing Office), 1234-1239.

Dickinson, K. R., 1970. Interpreting detrital modes from graywacke and arkose. J. Sediment. Petrol., 40:695-707.

Di Nocera, S., Ortolani, F., Torre, M., and Russo, B., 1979. Caratteristiche stratigrafiche e paleoambientali dei depositi altomiocenici nella zona di Falconara Albanese (Catena Costiera Calabra). Boll. Soc. Natur. Napoli, 83:1-29.

Fabbri, A., and Curzi, P. V., 1979. The Messinian of the Tyrrhenian Sea: seismic evidence and dynamic implications. G. Geol., 43:215248.

Finetti, I., and Del Ben, A., 1986. Geophysical study of the Tyrrhenian opening. Boll. Geof. Teor. Appl., 28:75-155.

Gazzi, P., 1966. Le arenarie del flysch sopracretaceo dell'Appennino modenese: correlazioni con il flysch di Monghidoro. Mineral. Petrogr. Acta, 16:69-97.

Girotti, 0., and Parotto, M., 1969. Mio-Pliocene di Ascoli Piceno. Atti Acc. Gioenia Sci. Nat. Catania, 7:127-174.

Hughes, A. D., and Whitehead, D., 1987. Glauconitization of detrital silica substrates in the Bantom Formation (Upper Eocene) of the Hampshire Basin, southern England. Sedimentology, 34:825-835.

Iaccarino, S., and Papani, G., 1979. Il Messiniano dell'Appennino Settentrionale dalla Val d'Arda alla Val Secchia: stratigrafia e rapporti con il substrato ed il Pliocene. Univ. Parma, Spec. Vol. dedicated to Sergio Venzo: $15-46$.

Kamp, J. J., and Nelson, C. S., 1987. Tectonic and sea-level controls on nontropical Neogene limestones in New Zealand. Geology, 15:610613.

Kastens, K. A., Mascle, J., et al., 1987. Proc. ODP, Init. Repts., 107: College Station, TX (Ocean Drilling Program).

Keller, G. H., and Lambert, D. N., 1972. Geotechnical properties of submarine sediments, Mediterranean Sea. In Stanley, D.H. (Ed.), The Mediterranean Sea: a natural sedimentation laboratory: Stroudsburg, Pa. (Dowden, Hutchinson and Ross), 401-415.

Lazzarotto, A., 1967. Geologia della zona compresa tra l'alta valle del Fiume Cornia ed il Torrente Pavone (Prov. di Pisa e Grosseto). Mem. Soc. Geol. It., 6:15-197.

Lazzarotto, A., and Mazzanti, R., 1978. Geologia dell'alta Val di Cecina. Boll. Soc. Geol. It., 95:1365-1487.

Lees, A., and Buller, A. T., 1972. Modern temperate-water and warmwater shelf carbonate sediments contrasted. Mar. Geol., 13:67-73.

Mascle, G., Sartori, R., et al., 1986. Nature du substratum de la marge sarde: resultats des forages de la campagne 107 du JOIDES Resolution (Ocean Drilling Program) (paper presented at the 30th CIESMM Congress, Palma de Mallorca, Spain). (Abstract).

Moussat, E., 1983. Evolution de la Mer Tyrrhenienne centrale et orientale et des ses marges septentrionales en relation avec la neotectonique dans l'Arc Calabrais. (Thése). Université Paris VI.
Nesteroff, W., 1973. Pétrographie des évaporites messiniennes de la Méditerranée. Comparaison des forages JOIDES-DSDP et des dépots du Bassin de Sicile. In Drooger, C. W. (Ed.), Messinian events in the Mediterranean. Kon. Nederl. Akad. van Wet.:111-123.

Odin, G. S., 1985. Significance of green particles (glaucony, Berthierine, Chlorite) in arenites. In Zuffa, G. G. (Ed.), Provenance of arenites. Dordrecht (Reidel), 279-307.

Orszag-Sperber, F., and Pilot, M. D., 1976. Grand traits du Néogene de Corse. Bull. Soc. Géol. Fr., 18:1183-1187.

Perrone, V., Torre, M., and Zuppetta, A., 1973. Il Miocene della Catena Costiera Calabra. Primo contributo: zona Diamante-BonifatiS.Agata d'Esaro (CS). Riv. It. Paleontol. Stratigr., 79:157-205.

Recq, M., Rehault, J. P., Steinmetz, L., and Fabbri, A., 1984. Amincissement de la croute et accretion au centre du bassin Tyrrhenien d'apres la sismique refraction. Mar. Geol., 55:411-428.

Rehault, J. P., Moussat, E., Mascle, J., and Sartori, R., 1985. Geodynamic evolution of the Tyrrhenian Sea. New data and drilling objectives (ODP Leg 107, Mediterranean) (paper presented at the 8th RCMNS Congress, Budapest). (Abstract).

Ricci Lucchi, F., 1973. Resedimented evaporites: indicators of slope instability and deep-water conditions in Peri-Adriatic Messinian (Apennines Foredeep Italy). In Drooger, C. W. (Ed.), Messinian events in the Mediterranean. Kon. Nederl. Akad. van Wet.:142-149. 1975. Miocene palaeogeography and basin analysis in the Periadriatic Apennines. In Squires, C. (Ed.), Geology of Italy, PESL, Tripoli, 2:129-136. , 1986. The Oligocene to Recent foreland basins of the northern Apennines. Spec. Publ. Int. Ass. Sediment., 8:105-139.

Ruggieri, G., 1958. Gli esotici neogenici della colata gravitativa della Val Marecchia. Atti Acc. Sci. Lett. Arti Palermo, 4/17:1-169.

1970. Note illustrative della Carta Geologica Italiana, F.109 Mercato Saraceno. Serv. Geol. Ital. Ercolano, 56 p.

Sartori, R., Mascle, G., and Amaudric du Chaffaut, S., 1987. A review of circum-Tyrrhenian regional geology. In Kastens, K., Mascle, J., et al., Proc. ODP, Init. Repts., 107: College Station, TX (Ocean Drilling Program), 37-63.

Sartori, R., et al., in press. Drillings of ODP Leg 107 in the Tyrrhenian Sea: tentative basin evolution compared to deformations in the surrounding chains. Atti Acc. Naz. Lincei Roma.

Sholle, P. A., 1971a. Sedimentology of fine-grained deep-water carbonate turbidites, Monte Antola Flysch (Upper Cretaceous), northern Apennines, Italy. Geol. Soc. Am. Bull., 82:629-658. , 1971b. Diagenesis of deep-water carbonate turbidites, Upper Cretaceous Monte Antola Flysch, northern Apennines, Italy. J. Sediment. Petrol., 41:233-250.

Skempton, A. W., 1953. The colloidal "activity" of clay. Proc. Third Intern. Conf. Soil Mechanics Found. Engineer. Switzerland, 57-61.

Tomadin, L., 1974. Les mineraux argileux dans les sediments actuels de la Mer Tyrrhenienne. Bull. Group Fr. Argiles, 26:219-228.

Vergnaud-Grazzini, C., 1985. Mediterranean Late Cenozoic Stable Isotope Record: Stratigraphic and Paleoclimatic Implications. In Stanley, D. J., and Wezel, F. C. (Eds.), Geological evolution of the Mediterranean Basin, 413-451.

Zachariasse, J. W., and Spaak, P., 1983. Middle Miocene to Pliocene paleoenvironmental reconstruction of the Mediterranean and adjacent Atlantic Ocean: planktonic foraminiferal record of Southern Italy. In Stanley, D. J., and Wezel, F. C. (Eds.), Geological evolution of the Mediterranean Basin, 91-110.

Zuffa, G. G., 1980. Hybrid arenites: their composition and classification. J. Sediment. Petrol., 50:21-29.

1985. Optical analyses of arenites: influence of methodology on compositional results. In Zuffa, G. G. (Ed.), Provenance of arenites. Dordrecht (Riedel), 279-307.

1987. Unravelling hinterland and offshore palaeogeography from deep-water arenites. In Leggett, J. K., and Zuffa, G. G. (Eds.), Marine Clastic Sedimentology Concepts and Case Studies. London (Graham and Trotman), 39-61.

Date of initial receipt: 5 January 1988

Date of acceptance: 17 January 1989

Ms 107B-128 